\title{
Duality, vector advection and the Navier-Stokes equations
}

\author{
Z. Brzeźniak and M. Neklyudov
}

Communicated by Charles Li, received July 29, 2008.

\begin{abstract}
In this article we show that three dimensional vector advection equation is self dual in certain sense defined below. As a consequence, we infer classical result of Serrin of existence of strong solution of Navier-Stokes equation. Also we deduce FeynmanKac type formula for solution of the vector advection equation and show that the formula is not unique i.e. there exist flows which differ from standard flow along which vorticity is conserved.
\end{abstract}

\section{CONTENTS}

1. Introduction 53

2. Notations and hypotheses 56

3. Duality 60

4. Formulae of Feynman-Kac Type. 65

5. Proofs of results from section $3 \quad 81$

References $\quad 92$

\section{Introduction}

The purpose of this paper is twofold. The first one is to establish a certain self-duality formula for a vector advection equation in the space $\mathbb{R}^{3}$. This formula can be understood as generalization of the helicity invariance for the Euler equations, see Corollary 3.13 and Remark 3.14. As a byproduct, see Corollary 3.13, we give a new proof of the classical result of Serrin [29] about the uniqueness of a weak solution to the Navier-Stokes equations

1991 Mathematics Subject Classification. 35Q30,60H30,76D05.

Key words and phrases. Navier-Stokes equations, Feynman Kac formula, vector advection.

The research of the second named authour was supported by an ORS award. 
(NSEs for short)

$$
\left\{\begin{aligned}
\frac{\partial u}{\partial t}+(u \cdot \nabla) u & =\nu \Delta u+\nabla p+f, \\
\operatorname{div} u & =0 \\
u(0) & =u_{0}
\end{aligned}\right.
$$

satisfying certain additional integrability condition. The second one, see Theorem 4.12, is to establish the existence of non-classical flows along with which the circulation of the solution of the vector advection equation is conserved in the mean. This problem seems to us important because it could potentially lead to the new a priori estimates of the solution of vector advection equation.

The importance of the vector advection equation stems from the fact that it appears in many different areas of hydrodynamics, e.g. the vorticity of a strong solution of the 3 -dimensional NSEs is its solution. Moreover, the major obstacle in proving the global existence of a strong solution to the NSEs is the appearance of the "vorticity stretching" term in the vector advection equation. It is necessary to underline that in a simpler case of the scalar advection equation, this conceptually important term is not present and therefore the self-duality and other properties described in this paper do not hold. Another application of the vector advection equation is the equation for magnetic field in MHD equations, see e.g. [24].

Let us describe briefly the main contributions of the paper.

In the first part of our paper we study the following vector advection equations

$$
\left\{\begin{aligned}
\frac{\partial F(t, x)}{\partial t} & =-\nu(A F)(t, x) \\
& -\left[\mathrm{P}\left(\left(\nabla F-\nabla F^{\perp}\right) v\right)\right](t, x)+f(t, x), x \in \mathbb{R}^{d}, \\
F(0, x) & =F_{0}(x), x \in \mathbb{R}^{d},
\end{aligned}\right.
$$

where $v:[0, \infty) \times \mathbb{R}^{d} \rightarrow \mathbb{R}^{d}$ is a given time-dependent vector field, $d=3, \mathrm{P}$ is the Helmholtz projection onto the divergence free vector fields and $A$ is the Stokes operator. As usual by $\mathbb{H}_{\text {sol }}^{k, 2}\left(\mathbb{R}^{d}\right), d \in \mathbb{N}$, we denote the space of all divergence free vector fields that belong to the Sobolev space $\mathbb{H}^{k, 2}\left(\mathbb{R}^{d}\right)$. Let us denote by $\mathcal{T}_{T}^{v}$ the transport operator along $v$, i.e. $\mathcal{T}_{t}^{v} F_{0}=F(t)$, for $t \geq 0$, where $f$ is the unique solution to problem (1.2). The main result here is Theorem 3.7 in which we formulate the following self-duality formula.

$$
\left(\operatorname{curl} F_{0}, \mathcal{T}_{T}^{S_{T} v} G_{0}\right)_{H}=\left(\operatorname{curl} \mathcal{T}_{T}^{v} F_{0}, G_{0}\right)_{H}, F_{0} \in \operatorname{curl}^{-1}(H), G_{0} \in H,
$$

where $S_{T}$ is the time reversal operator, i.e. $\left(S_{T} v\right)(t)=-v(T-t), t \in[0, T]$. The self-duality formula (1.3) allows us to deduce certain properties of the operator $\mathcal{T}_{T}^{v}$. In particular in Corollary 3.10 we show that the $\mathcal{L}\left(\mathbb{H}_{\text {sol }}^{k, 2}, \mathbb{H}_{\text {sol }}^{k, 2}\right)-$ norm of $\mathcal{T}_{T}^{v}$ is equal to its $\mathcal{L}\left(\mathbb{H}_{\text {sol }}^{1-k, 2}, \mathbb{H}_{\text {sol }}^{1-k, 2}\right)$ - norm. Moreover, in Corollary 3.12, we prove that the space $\mathcal{L}\left(\mathbb{H}_{\text {sol }}^{\frac{1}{2}, 2}, \mathbb{H}_{\text {sol }}^{\frac{1}{2}, 2}\right)$ is in a certain sense optimal for $\mathcal{T}_{T}^{v}$.

The main result in the second part of the paper, Theorem 4.12, is about a certain nonclassical Feynman-Kac type formula for the solutions of the vector advection equation (1.2) in two dimensions. We show that if the divergence free vector field $v$ is time-independent and sufficiently regular, then the stochastic flow of diffeomorphisms of $\mathbb{R}^{2} X_{s}(t ; \cdot), 0 \leq$ 
$s \leq t \leq T$, corresponding to the following $\operatorname{SDE}$ on $\mathbb{R}^{2}$,

$$
\left\{\begin{aligned}
d X_{s}(t ; x) & =\sqrt{2 \nu} \sigma_{1}\left(X_{s}(t ; x)\right) d W(t), 0 \leq s \leq t \leq T, \\
X_{s}(s ; x) & =x .
\end{aligned}\right.
$$

where, with a function $\phi: \mathbb{R}^{2} \rightarrow \mathbb{R}$ such that ${ }^{1} v=\nabla^{\perp} \phi$,

$$
\sigma_{1}(x)=\left(\begin{array}{cc}
\cos \frac{\phi(x)}{\nu} & -\sin \frac{\phi(x)}{\nu} \\
\sin \frac{\phi(x)}{\nu} & \cos \frac{\phi(x)}{\nu}
\end{array}\right), x \in \mathbb{R}^{2},
$$

has the following properties: (i) its one-point motion is a Brownian Motion and (ii) the circulation along it of the solution of the two dimensional vector advection equation (1.2), i.e. with $d=2$, is a martingale. This flow seems to be of interest on its own because the stream function $\phi$ naturally arise in its construction.

The question of the existence of an analogous flow in the three dimensional case remains open, see Question (4.24) for details.

It should be noticed here that a similar construction does not work for the scalar advection equation because in this case the Feynman-Kac type formula depends only upon the law of the flow itself and not upon the law of the gradient of the flow. Also we would like to point out that the main obstacle in getting a'priori estimates for solutions of vector advection equation (in particular, for vorticity of the solution to the 3-D NSEs) is lack of an estimate for the gradient of the flow. Therefore, in connection with this result, a natural question is whether it is possible to choose the optimal flow for which gradient is bounded?

The main idea behind our approach to the Feynman-Kac type formula for solutions of the vector advection equation is that in the case with viscosity equal to 0 , the conservation law of circulation, known also as Kelvin-Noether Theorem, holds. In the case of positive viscosity we are able to find an analog of this conservation law. The Feynman-Kac formula is then an immediate consequence of that result. This idea has been used before in the papers [25] and [26] (though with quite sketchy proofs). In the latter paper, see Theorem 5 and Example 1, the Feynman-Kac formula for the solution of vector advection equation without incompressibility condition has been derived. A somewhat similar idea has been also explored independently by Constantin and Iyer in [6], but see also Flandoli et al. [5] for a different approach. Moreover, Flandoli et al. [5] proved Feynman-Kac formula for more general systems of parabolic PDEs. However, we would like to point out that in all of the articles mentioned above only the "standard" stochastic flow corresponding to the following SDE

$$
\begin{aligned}
\left.d X_{s}(t ; x)\right) & =v\left(t, X_{s}(t ; x)\right) d t+\sqrt{2 \nu} d W(t), t \in[s, T], \\
X_{s}(s ; x) & =x .
\end{aligned}
$$

has been used and, correspondingly, the problems discussed here does not appear in their framework.

One possible application of Theorem 4.12 is the extension of Le Jan and Raimond's theory of statistical solutions of the scalar advection equations, see [18], to the $2 \mathrm{D}$ vector advection case. Indeed, Le Jan, Raimond theory defines statistical solution $X_{s}(t ; x)$ of SDE (1.5) (corresponding to a solution of scalar advection equation in a natural way) with velocity $v$ given by

$$
d v^{i}(t, x)=\sum_{k=1}^{\infty} \sigma_{k}^{i}(x) d W(t)^{k}, x \in \mathbb{R}^{n}, t \geq 0, i=1, \ldots, n
$$

\footnotetext{
${ }^{1}$ Such $\phi$ exists because $\operatorname{div} v=0$.
} 
where $\sigma_{k}^{i}(\cdot)$ are Hölder continuous and $\left\{W(t)^{k}\right\}_{k=1}^{\infty}$ is a family of i.i.d. Wiener processes. In the case of the $2 \mathrm{D}$ vector advection, Theorem 4.12 implies that we don't need to define process $X_{s}(t ; x)$ (It is just Brownian motion!). We only need to show that the linear equation (4.21) for the gradient of the flow $\nabla X_{s}(t ; x)$ has a strong solution. At this moment, there appears certain difficulty with the definition of the right hand side of equation (4.21) for irregular vector field $v$ of the form (1.6). We are of the impression that the white noise calculus could be of some help here.

Finally, the idea of generalization of the conservation laws has been extensively studied in physical literature, where it is called statistical integral of motion or zero mode, see e.g. the survey [9, part II.E, p.932], and references therein.

Note: After we had proved Corollary 4.3 we became aware that independently of us a similar result was proved recently by Constantin and Iyer in [6].

Acknowledgments. We would like to thank T. Komorowski and B. Gołdys for their useful remarks, in particular to the former one for informing us about the work by Constantin and Iyer [6]. The present article derives from work done as part of the $\mathrm{Ph}$. D. thesis of the second named author at the University of York, while supported by the ORS award, University of York scholarship and, later, by an ARC Discovery project DP0558539. The research of the first named author was supported by an by the EPSRC grant EP/E01822X/1 and the ARC Discovery grant DP0663153.

\section{Notations and hypotheses}

Let $D$ be either $\mathbb{R}^{d}$ or an open, bounded and connected set in $\mathbb{R}^{d}$. In the latter case, we assume that the boundary $\Gamma=\partial D$ of $D$ is of $C^{3}$ class and we denote by $\vec{n}$ the outer normal vector field to the boundary $\Gamma$. We denote by $C^{\infty}\left(D, \mathbb{R}^{n}\right)$ the space of infinitely differentiable functions from $D$ to $\mathbb{R}^{n}$ and by $C_{0}^{\infty}\left(D, \mathbb{R}^{n}\right)$ the subspace of those functions belonging to $C^{\infty}\left(D, \mathbb{R}^{n}\right)$ which have a compact support. Finally, let us denote

$$
\mathcal{D}(D)=\left\{f \in C_{0}^{\infty}\left(D, \mathbb{R}^{d}\right): \operatorname{div} f=0\right\} .
$$

For $k \in \mathbb{N}$ and $p \in[1, \infty)$, let $H_{0}^{k, p}\left(D, \mathbb{R}^{n}\right)$, respectively $H^{k, p}\left(D, \mathbb{R}^{n}\right)$, be the completion of $C_{0}^{\infty}\left(D, \mathbb{R}^{n}\right)$, respectively $C^{\infty}\left(D, \mathbb{R}^{n}\right)$, with respect to norm

$$
\|f\|_{k, p}=\left(\sum_{l=0}^{k} \sum_{|\alpha| \leq l} \int_{D}\left|D^{\alpha} f(x)\right|_{\mathbb{R}^{n}}^{p} d x\right)^{1 / p} .
$$

We will use the following notation

$$
\begin{aligned}
H^{k, p}(D) & =H^{k, p}(D, \mathbb{R}), & H_{0}^{k, p}(D) & =H_{0}^{k, p}(D, \mathbb{R}), \\
\mathbb{H}^{k, p}(D) & =H^{k, p}\left(D, \mathbb{R}^{d}\right), & \mathbb{H}_{0}^{k, p}(D) & =H_{0}^{k, p}\left(D, \mathbb{R}^{d}\right), \\
\mathbb{H}^{k}(D) & =\mathbb{H}^{k, 2}(D), & \mathbb{L}^{p}(D) & =H^{0, p}\left(D, \mathbb{R}^{d}\right) .
\end{aligned}
$$

Finally, let us denote

$$
\begin{aligned}
H & =\left\{f \in \mathbb{L}^{2}(D): \operatorname{div} f=0,\left.(f \cdot \vec{n})\right|_{\Gamma}=0\right\}, \\
V & =\mathbb{H}_{0}^{1,2}(D) \cap H .
\end{aligned}
$$

Equipped with the norm $\|\cdot\|_{0,2}, H$ is a Hilbert space. Similarly, $V$ is a Hilbert space when equipped with the norm $\|\cdot\|_{1,2}$. The norms in $H$ and $V$ will be denoted by $|\cdot|$ and $\|\cdot\|$. See also [33, pp. 9-15] for the definition and different characterizations of the spaces $H$ and $V$. 
By $\mathbb{H}_{\text {sol }}^{k, p}(D)$ we will denote the completion of $\mathcal{D}(D)$ w.r.t. the norm $\|\cdot\|_{k, p}$. We will often write $\mathbb{H}_{\text {sol }}^{k, p}$ instead of $\mathbb{H}_{\text {sol }}^{k, p}\left(\mathbb{R}^{3}\right)$. We also denote by $\mathbb{H}_{h, \text { sol }}^{k, p}$ the completion of $\mathcal{D}\left(\mathbb{R}^{3}\right)$ w.r.t. the homogeneous norm

$$
\|f\|_{k, p}^{h}=\left(\int_{\mathbb{R}^{3}}\left|\operatorname{curl}^{k} f\right|_{\mathbb{R}^{3}}^{p} d x\right)^{1 / p}, k \in \mathbb{N}, \in[1, \infty) .
$$

Let us also denote $\mathbb{H}_{h, \text { sol }}^{-k, 2}=\left(\mathbb{H}_{h, \text { sol }}^{k, 2}\right)^{*}, k \in \mathbb{N}$ and define the spaces with fractional order via the complex interpolation, i.e.

$$
\mathbb{H}_{h, \text { sol }}^{\alpha, p}=\left[\mathbb{H}_{h, \text { sol }}^{[\alpha], p}, \mathbb{H}_{h, \text { sol }}^{[\alpha]+1, p}\right]_{\alpha-[\alpha]}, \alpha \in \mathbb{R},
$$

where $[\cdot, \cdot]_{\beta}$ is a complex interpolation space of order $\beta$.

Let $\mathrm{P}: \mathbb{H}^{k, p}(D) \rightarrow \mathbb{H}_{\text {sol }}^{k, p}(D)$ be the Helmholtz projection onto the divergence-free vector fields, see [11] or [33].

From now on we consider the case $d=3$. By $\times$ we will denote the vector product in $\mathbb{R}^{3}$. We will often use the following properties of the vector product.

$$
\begin{array}{r}
(a \times b, c)_{\mathbb{R}^{3}}=(a, b \times c)_{\mathbb{R}^{3}} \\
|a \times b|_{\mathbb{R}^{3}} \leq|a|_{\mathbb{R}^{3}}|b|_{\mathbb{R}^{3}} .
\end{array}
$$

We will identify the dual $H^{\prime}$ with $H$ and so we can assume that $H \subset V^{\prime}$. In particular,

$$
V \subset H \cong H^{\prime} \subset V^{\prime}
$$

is Gelfand triple. We will need the following results borrowed from the monograph [19] by Lions and Magenes, see Theorem 3.1, p. 19 and Proposition 2.1, p. 18.

Lemma 2.1. Suppose that $\mathcal{V} \subset \mathcal{H} \subset \mathcal{V}^{\prime}$ is a Gelfand triple with the duality relation

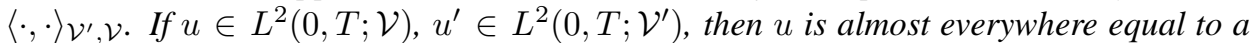
continuous function from $[0, T]$ into $\mathcal{H}$ and we have the following equality, which holds in the scalar distribution sense on $(0, T)$ :

$$
\frac{d}{d t}|u|^{2}=2\left\langle u^{\prime}, u\right\rangle .
$$

As a consequence we have the following result.

COROLlary 2.2. If $f, g \in L^{2}(0, T ; \mathcal{V})$ with $f^{\prime}, g^{\prime} \in L^{2}\left(0, T ; \mathcal{V}^{\prime}\right)$ then $(f, g)_{\mathcal{H}}$ is almost everywhere equal to weakly differentiable function and

$$
\frac{d}{d t}(f, g)_{\mathcal{H}}=\left\langle f^{\prime}, g\right\rangle_{\mathcal{V}^{\prime}, \mathcal{V}}+\left\langle f, g^{\prime}\right\rangle_{\mathcal{V}^{\prime}, \mathcal{V}}
$$

We also recall the following result from [19], see Theorem 4.1, p. 238 and Remark 4.3, p. 239.

THEOREM 2.3. Assume that

$$
A \in L^{\infty}\left([0, T], \mathcal{L}\left(\mathcal{V}, \mathcal{V}^{\prime}\right)\right)
$$

satisfies the following coercivity condition. There exist $\alpha>0$ and $\lambda \in \mathbb{R}$ such that

$$
\langle A(t) u, u\rangle_{\mathcal{V}^{\prime}, \mathcal{V}} \geq \alpha|u|_{\mathcal{V}}^{2}+\lambda|u|_{\mathcal{H}}^{2}, u \in \mathcal{V} .
$$

Then for all $u_{0} \in \mathcal{H}$ and $f \in L^{2}\left(0, T ; \mathcal{V}^{\prime}\right)$ the problem

$$
\left\{\begin{array}{rl}
\frac{d u}{d t}+A u & =f \\
u(0) & =u_{0}
\end{array},\right.
$$


has unique solution $u \in L^{2}(0, T ; \mathcal{V})$ such that $u^{\prime} \in L^{2}\left(0, T ; \mathcal{V}^{\prime}\right)$. Moreover, this unique solution u satisfies the following inequality

$$
|u|_{H}^{2}(t)+\alpha \int_{0}^{t}|u(s)|_{V}^{2} d s \leq(1+2 \lambda t) e^{2 \lambda t}\left(\left|u_{0}\right|_{H}^{2}+\frac{1}{4 \alpha} \int_{0}^{t}|f|_{V^{\prime}}^{2} d s\right), t \in[0, T] .
$$

We will also need the following result.

Proposition 2.4. Assume that an operator $A \in \mathcal{L}\left(\mathcal{V}, \mathcal{V}^{\prime}\right)$ satisfies the coercivity condition (2.6). Let us denote $D(A)=\{x \in \mathcal{H} \mid A x \in \mathcal{H}\}$. Then for all $f \in L^{2}(0, T ; \mathcal{H})$ and $u_{0} \in \mathcal{V}$ there exists a unique solution $u \in L^{2}(0, T ; D(A)) \cap C([0, T] ; \mathcal{V})$ of the problem:

$$
\left\{\begin{array}{rl}
\frac{d u}{d t}+\nu A u & =f \\
u(0) & =u_{0}
\end{array},\right.
$$

and it satisfies $u^{\prime} \in L^{2}(0, T ; \mathcal{H})$. Moreover, there exists a constant $C=C(\lambda, T, \nu)$ (independent of $u_{0}$ and $f$ ) such that

$$
\left|u^{\prime}\right|_{L^{2}(0, T ; \mathcal{H})}^{2}+\nu^{2}|u|_{L^{2}(0, T ; D(A))}^{2} \leq C\left(|f|_{L^{2}(0, T ; \mathcal{H})}^{2}+\left|u_{0}\right|_{\mathcal{V}}^{2}\right) .
$$

Proof of Proposition 2.4. It follows from Theorem 3.6.1 p.76 of [32] that $-A$ generates an analytic semigroup in $\mathcal{H}$. Therefore, the existence and the uniqueness of solution $u$ follows from Theorem 3.2 p.22 of [20]. It remains to show the inequality (2.8). Let us define a Banach space $X=\left\{u \in L^{2}(0, T ; D(A)): u^{\prime} \in L^{2}(0, T ; H)\right\}$ and a bounded linear operator $\left.\mathcal{Q}: X \ni u \mapsto\left(u(0), u^{\prime}+A u\right) \in V \times L^{2}(0, T ; H)\right)$. Since $\mathcal{Q}$ is a bijection, according to the Open Mapping Theorem, there exists the inverse continuous operator $\mathcal{Q}^{-1}$, i.e. $\mathcal{Q}^{-1} \in \mathcal{L}\left(V \times L^{2}(0, T ; H), X\right)$. Hence the inequality (2.8) holds.

DEFINITION 2.5. Let us define a bilinear form $\tilde{a}: V \times V \rightarrow \mathbb{R}$ by

$$
\tilde{a}(u, v)=\sum_{i, j=1}^{3} \int_{D} \nabla_{i} u^{j} \nabla_{i} v^{j} d x, u, v \in V .
$$

Lemma 2.6. The form $\tilde{a}: V \times V \rightarrow \mathbb{R}^{1}$ is positive, bilinear, continuous and symmetric.

Proof. Proof is omitted.

It follows from Lemma 2.6 and the Lax-Milgram Theorem that for any $f \in V^{\prime}$ there exists unique $u \in V$ such that

$$
\tilde{a}(u, v)+\lambda(u, v)=\langle f, v\rangle_{V^{\prime}, V}, v \in V .
$$

Definition 2.7. Define $A \in \mathcal{L}\left(V, V^{\prime}\right)$ by an identity

$$
\tilde{a}(u, v)=\langle A u, v\rangle_{V, V^{\prime}}, u, v \in V .
$$

REMARK 2.8. The operator $A$ defined above is often called the Stokes operator.

COROLlary 2.9. The operator A defined in Definition 2.7 is self-adjoint and positive definite.

PROOF. Follows from the symmetry of the form $\tilde{a}$, Theorem 2.2.3, Remark 2.2.1, p.29 of [32]. 
DEFINITION 2.10. Let us define trilinear form $\tilde{b}: C_{0}^{\infty}(D) \times \mathcal{D} \times \mathcal{D} \rightarrow \mathbb{R}$ by

$$
\tilde{b}(v, f, \phi)=\langle\mathrm{P}(v \times \operatorname{curl} f), \phi\rangle_{V^{\prime}, V},(v, f, \phi) \in C_{0}^{\infty}(D) \times \mathcal{D} \times \mathcal{D} .
$$

LEMMA 2.11. For any $\delta$ there exists $C_{\delta}>0$ such that for all $\varepsilon>0$ and all $(v, f, \phi) \in$ $C_{0}^{\infty}(D) \times \mathcal{D} \times \mathcal{D}$

(2.11) $|\tilde{b}(v, f, \phi)|^{2} \leq|f|_{V}^{2}|\phi|_{V}^{2}\left(\varepsilon^{1+\delta / 3}+\frac{C_{\delta}}{\varepsilon^{1+3 / \delta}}|v(t)|_{\mathbb{L}^{3+\delta}(D)}^{2+\frac{6}{\delta}}\right)$,

(2.12) $|\tilde{b}(v, f, \phi)| \leq \frac{1}{2}\|f\|_{V}^{2}+\frac{1}{2}\left(\varepsilon^{1+\delta / 3}\|\phi\|_{V}^{2}+\frac{C_{\delta}}{\varepsilon^{1+3 / \delta}}|v(t)|_{\mathbb{L}^{3+\delta}(D)}^{2+\frac{6}{\delta}}|\phi|_{H}^{2}\right)$.

Moreover, if we assume that $f \in D(A)$, then for any $\phi \in V$ the following inequality holds

$$
|\tilde{b}(v, f, \phi)|^{2} \leq|\phi|_{H}^{2}\left(\varepsilon^{1+\delta / 3}\|f\|_{D(A)}^{2}+\frac{C_{\delta}}{\varepsilon^{1+3 / \delta}}|v|_{\mathbb{L}^{3+\delta}(D)}^{2+\frac{6}{\delta}}|f|_{V}^{2}\right)
$$

To prove Lemma 2.11 we will need the following auxiliary result.

LEMMA 2.12. For any $\delta$ there exists $C_{\delta}>0$ such that for all $\varepsilon>0$

$$
\|f \times g\|_{\mathbb{L}^{2}(D)}^{2} \leq \varepsilon^{1+\delta / 3}\|f\|_{V}^{2}+\frac{C_{\delta}}{\varepsilon^{1+3 / \delta}}|g|_{\mathbb{L}^{3+\delta}(D)}^{2+\frac{6}{\delta}}|f|_{H}^{2}, f \in V, g \in H .
$$

Proof of Lemma 2.12. Let us $p=3-\frac{2 \delta}{1+\delta}, q=\frac{3+\delta}{2}, \theta=\frac{3}{3+\delta}$. Then $\frac{1}{p}+\frac{1}{q}=1$ and therefore by the inequality (2.2), the Hölder inequality, the Gagliardo-Nirenberg inequality (see Theorem 9.3, p.24 in [10]) and from the Young inequality we infer the following train of inequalities

$$
\begin{aligned}
\|f \times g\|_{\mathbb{L}^{2}(D)}^{2} & \leq \int_{D}|f|^{2}|g|^{2} d x \leq|f|_{\mathbb{L}^{2 p}(D)}^{2}|g|_{\mathbb{L}^{2 q}(D)}^{2} \\
\leq\left(\|f\|_{V}^{\theta}|f|_{H}^{1-\theta}\right)^{2}|g|_{\mathbb{L}^{2 q}(D)}^{2} & \leq \varepsilon^{1+\delta / 3}\|f\|_{V}^{2}+\frac{C_{\delta}}{\varepsilon^{1+3 / \delta}}|g|_{\mathbb{L}^{3+\delta}(D)}^{2+\frac{6}{\delta}}|f|_{H}^{2} .
\end{aligned}
$$

Proof of Lemma 2.11. Let us fix $(v, f, \phi) \in C_{0}^{\infty}(D) \times \mathcal{D} \times \mathcal{D}$. Then by equality (2.1), and Lemma 2.12 we have

$$
\begin{aligned}
|\tilde{b}(v, f, \phi)|^{2} & =\left|\langle v(t) \times \phi, \operatorname{curl} f\rangle_{V^{\prime}, V}\right|^{2} \\
& \leq|\operatorname{curl} f|_{H}^{2}|v(t) \times \phi|_{H}^{2} \\
& \leq\|f\|_{V}^{2}\left(\varepsilon^{1+\delta_{0} / 3}\|\phi\|_{V}^{2}+\frac{C_{\delta_{0}}}{\varepsilon^{1+3 / \delta_{0}}}|v(t)|_{\mathbb{L}^{3+\delta_{0}(D)}}^{2+\frac{6}{\delta_{0}}}|\phi|_{H}^{2}\right) \\
& \leq|f|_{V}^{2}|\phi|_{V}^{2}\left(\varepsilon^{1+\delta_{0} / 3}+\frac{C_{\delta_{0}}}{\varepsilon^{1+3 / \delta_{0}}}|v(t)|_{\mathbb{L}^{3+\delta_{0}(D)}}^{2+\frac{6}{\delta_{0}}}\right) .
\end{aligned}
$$

Similarly,

$$
\text { (2.16) } \begin{aligned}
|\tilde{b}(v, f, \phi)| & =\left|\langle v(t) \times \phi, \operatorname{curl} f\rangle_{V^{\prime}, V}\right| \leq|\operatorname{curl} f|_{H}|v(t) \times \phi|_{H} \\
& \leq \frac{1}{2}\|f\|_{V}^{2}+\frac{1}{2}|v(t) \times \phi|_{H}^{2} \\
& \leq \frac{1}{2}\|f\|_{V}^{2}+\frac{1}{2}\left(\varepsilon^{1+\delta_{0} / 3}\|\phi\|_{V}^{2}+\frac{C_{\delta_{0}}}{\varepsilon^{1+3 / \delta_{0}}}|v(t)|_{\mathbb{L}^{3+\delta_{0}(D)}}^{2+\frac{6}{\delta_{0}}}|\phi|_{H}^{2}\right),
\end{aligned}
$$

and

$$
\begin{aligned}
|\tilde{b}(v, f, \phi)|^{2} & =\left|\langle v(t) \times \operatorname{curl} f, \phi\rangle_{V^{\prime}, V}\right|^{2} \leq|\phi|_{H}^{2}|v(t) \times \operatorname{curl} f|_{H}^{2} \\
& \leq|\phi|_{H}^{2}\left(\varepsilon^{1+\delta / 3}|f|_{D(A)}^{2}+\frac{C_{\delta}}{\varepsilon^{1+3 / \delta}}|v|_{\mathbb{L}^{3+\delta}(D)}^{2+\frac{6}{\delta}}|f|_{V}^{2}\right)
\end{aligned}
$$


Fix $\delta_{0}>0$. It follows from inequality (2.11) that the trilinear form $\tilde{b}$ is continuous with respect to the $L^{3+\delta_{0}}(D) \times V \times V$ topology. Therefore, there exist continuous trilinear form $b: L^{3+\delta_{0}}(D) \times V \times V \rightarrow \mathbb{R}$ such that

$$
\left.b(\cdot, \cdot, \cdot)\right|_{C_{0}^{\infty}(D) \times \mathcal{D} \times \mathcal{D}}=\tilde{b} .
$$

Moreover,

$$
b(v, f, \phi)=-(v \times \phi, \operatorname{curl} f)_{H},(v, f, \phi) \in L^{3+\delta_{0}}(D) \times V \times V .
$$

Indeed, the form on the left hand side of equality (2.18) is equal to the form on the right hand side of equality (2.18) for $(v, f, \phi) \in C_{0}^{\infty}(D) \times \mathcal{D} \times \mathcal{D}$ and both forms are continuous in $L^{3+\delta_{0}}(D) \times V \times V$.

DEFINITION 2.13. Let us define a bilinear operator $B: L^{3+\delta_{0}}(D) \times V \rightarrow V^{\prime}$ by

$$
\langle B(v, f), \phi\rangle_{V^{\prime}, V}=b(v, f, \phi), v \in L^{3+\delta_{0}}(D), f \in V, \phi \in V .
$$

COROLlary 2.14. Assume that $\delta_{0}>0$. Then there exists a constant $C_{\delta_{0}}>0$ independent such that

$$
|B(v, f)|_{V^{\prime}}^{2} \leq\|f\|_{V}^{2}\left(\varepsilon^{1+\delta_{0} / 3}+\frac{C_{\delta_{0}}}{\varepsilon^{1+3 / \delta_{0}}}|v(t)|_{\mathbb{L}^{3+\delta_{0}(D)}}^{2+\frac{6}{\delta_{0}}}\right),(v, f) \in L^{3+\delta_{0}}(D) \times V .
$$

Moreover, if $(v, f) \in L^{3+\delta_{0}}(D) \times D(A)$ then $B(v, f) \in H$ and

$$
|B(v, f)|_{H}^{2} \leq\left(\varepsilon^{1+\delta_{0} / 3}\|f\|_{D(A)}^{2}+\frac{C_{\delta_{0}}}{\varepsilon^{1+3 / \delta_{0}}}|v|_{\mathbb{L}^{3+\delta_{0}(D)}}^{2+\frac{6}{\delta}}|f|_{V}^{2}\right)
$$

Proof of Corollary 2.14. Proof immediately follows from Lemma 2.11.

\section{Duality}

Assume that $F_{0} \in H, f \in L^{2}\left(0, T ; V^{\prime}\right)$. We consider the following two problems:

$$
\begin{aligned}
\frac{\partial F}{\partial t} & =-\nu A F-B(v(t), F)+f, \\
F(0) & =F_{0}, \\
\frac{\partial G}{\partial t} & =-\nu A G-\operatorname{curl}(v(t) \times G)+f, \\
G(0) & =G_{0} .
\end{aligned}
$$

DEFINITION 3.1. We will call an element $F$, respectively $G$, of $L^{2}(0, T ; V) \cap$ $L^{\infty}(0, T ; H) \cap C\left([0, T] ; H_{\mathrm{w}}\right)$, where $H_{\mathrm{w}}$ is equal to $H$ endowed with the weak topology, a solution of problem (3.1-3.2), resp. (3.3-3.4), iff F, resp. G, satisfies equation (3.1), resp. problem (3.3), in the distribution sense and $F$, resp. G, satisfies (3.2), resp. (3.4) as elements of $C\left([0, T] ; H_{\mathrm{w}}\right)$.

In the next two Propositions we will deal with the existence and regularity results for solutions of problems (3.1-3.2) and (3.3-3.4). These results are probably known, but since we have been unable to find them (in the form we need) in the literature, we have decided to present them for the sake of the completeness of the paper.

Proposition 3.2. Suppose that $\left(F_{0}, f\right) \in H \times L^{2}\left(0, T ; V^{\prime}\right)$ and

$$
v \in \bigcup_{\delta_{0}>0} L^{2+\frac{6}{\delta_{0}}}\left(0, T ; \mathbb{L}^{3+\delta_{0}}(D)\right) .
$$

Then 
(i) there exists the unique solution $F$ of problem (3.1-3.2) and for each $\delta_{0}>0$ there exists a constant $K_{1}=K_{1}\left(|v|_{L^{2+\frac{6}{\delta_{0}}}\left(0, T ; \mathbb{L}^{3+\delta_{0}}(D)\right)}, \nu\right)$ independent of $\left(F_{0}, f\right)$ such that $F$ satisfies the following inequality

$$
\begin{array}{ll}
|F(t)|_{H}^{2} & +\nu \int_{0}^{t}\|F(s)\|_{V}^{2} d s \\
& \leq K_{1}\left(\int_{0}^{t}|v(s)|_{\mathbb{L}^{3+\delta_{0}}}^{2+6 / \delta_{0}} d s, \nu\right)\left(\left|F_{0}\right|_{H}^{2}+\frac{C}{\nu} \int_{0}^{t}|f(s)|_{V^{\prime}}^{2} d s\right), t \in[0, T] .
\end{array}
$$

Furthermore, $F \in C\left([0, T], V^{\prime}\right)$ and $F^{\prime} \in L^{1+\frac{3}{2 \delta_{0}+3}}\left(0, T ; V^{\prime}\right)$. Moreover, if $v$ satisfies the following, stronger than (3.5), condition

$$
v \in L^{\infty}\left(0, T ; \mathbb{L}^{3+\delta_{0}}(D)\right) \text { for some } \delta_{0}>0
$$

then $F^{\prime} \in L^{2}\left(0, T ; V^{\prime}\right)$.

(ii) If in addition $\left(F_{0}, f\right) \in V \times L^{2}(0, T ; H)$ and the condition (3.7) is satisfied, then $F \in C([0, T], V) \cap L^{2}(0, T ; D(A))$.

(iii) Assume that $n \in \mathbb{N}$. Suppose $f^{(n)} \in L^{2}(0, T ; H)$, there exists $\delta_{0}>0$ such that $v \in$ $C^{n-1}\left(0, T ; \mathbb{L}^{3+\delta_{0}}(D)\right), v^{(n)} \in L^{\infty}\left(0, T ; \mathbb{L}^{3+\delta_{0}}(D)\right)$ and $g_{k} \in V$, for $k=0,1, \ldots, n$, where sequence $\left\{g_{k}\right\}_{k=0}^{\infty}$ is defined by formula ${ }^{2}$

$$
\begin{aligned}
g_{m} & \left.=-\nu A g_{m-1}-\sum_{k=0}^{m-1} B\left(v^{(m-k-1)}\right)(0), g_{k}\right)+f^{m-1}(0), m=1, \ldots, n \\
g_{0} & =F_{0} .
\end{aligned}
$$

Then $F \in C^{n}([0, T], V)$.

REMARK 3.3. We should notice that on the one hand, our class $\bigcup_{\delta>0} L^{2+\frac{6}{\delta_{0}}}\left(0, T ; \mathbb{L}^{3+\delta_{0}}(D)\right)$ is the Serrin regularity class. Indeed, if $r=2+\frac{6}{\delta_{0}}$, $s=3+\delta_{0}$ then $\frac{2}{r}+\frac{3}{s}=1$. Therefore, any weak solution of the NSEs belonging to this class is a strong solution. On the other hand, we have been unable to prove that under the assumption (3.5) a solution $F$ of problem (3.1-3.2) is such that $F^{\prime} \in L^{2}\left(0, T, V^{\prime}\right)$. A problem that arises here is similar to the problem whether a weak solution $u$ of the NSEs, see [33], p. 191 Problem 3.2 and Theorem 3.1, satisfies $u^{\prime} \in L^{2}\left(0, T ; V^{\prime}\right)$.

For the second equation we have:

PROPOSITION 3.4. Suppose that a time dependent vector field $v$ satisfies the assumption (3.5). Then

(i) for every $\left(F_{0}, f\right) \in H \times L^{2}\left(0, T ; V^{\prime}\right)$ there exists unique solution $G$ of the problem (3.3-3.4) such that $G^{\prime} \in L^{2}\left(0, T ; V^{\prime}\right)$ and for each $\delta_{0}>0$ there exists a constant

\footnotetext{
${ }^{2}$ It is easy to see that formally system (3.1-3.2) uniquely defines $F^{(k)}(0)$. Indeed, if we formally put $t=0$ in the system we immediately get expression for $F^{\prime}(0)$ through known parameters. Similarly, differentiating equation 3.1 w.r.t. time we get recurrent formula (3.8) for $F^{(k)}(0), k \in \mathbb{N}$. So, the condition $g_{k} \in V$ is compatibility condition.
} 
$K_{1}\left(\int_{0}^{t}|v(s)|_{\mathbb{L}^{3+\delta_{0}}}^{2+6 / \delta_{0}} d s, \nu\right)$ such that $G$ satisfies the following inequality for $t \in[0, T]$,

$|G(t)|_{H}^{2}+\nu \int_{0}^{t}\|G(s)\|_{V}^{2} d s$

$$
\leq K_{1}\left(\int_{0}^{t}|v(s)|_{\mathbb{L}^{3+\delta_{0}}}^{2+6 / \delta_{0}} d s, \nu\right)\left(\left|G_{0}\right|_{H}^{2}+\frac{C}{\nu} \int_{0}^{t}|f(s)|_{V^{\prime}}^{2} d s\right), t \in[0, T] .
$$

(ii) If in addition $v \in L^{2}(0, T, V)$ and $\left(F_{0}, f\right) \in V \times L^{2}(0, T ; H)$, then the solution $G$ from part (i) satisfies $G \in C([0, T], V) \cap L^{2}(0, T ; D(A))$.

(iii) Fix $n \in \mathbb{N}$. If $f^{(n)} \in L^{2}(0, T ; H)$, there exists $\delta_{0}>0$ such that $v \in$ $C^{n-1}\left(0, T ; \mathbb{L}^{3+\delta_{0}}(D)\right), v^{(n)} \in L^{\infty}\left(0, T ; \mathbb{L}^{3+\delta_{0}}(D)\right) \cap L^{2}(0, T, V)$ and $l_{k} \in V$, for $k=0,1, \ldots, n$, where sequence $\left\{l_{k}\right\}_{k=0}^{\infty}$ is defined by formula

$$
\begin{aligned}
l_{m}= & -\nu A l_{m-1}-\sum_{k=0}^{m-1} \operatorname{curl}\left(v^{(m-k-1)}(0) \times l_{k}\right) \\
& +f^{m-1}(0), m=1, \ldots, n \\
l_{0}= & G_{0} .
\end{aligned}
$$

Then $G \in C^{n}([0, T], V)$.

Corollary 3.5. Assume that $F_{0} \in H, f, v \in C^{\infty}([0, T] ; H)$. If for each $k \in \mathbb{N}, v^{(k)}$ satisfies the condition (3.5), then the solution of the problem (3.1-3.2) is in $C^{\infty}((0, T] \times D)$.

Proof of Corollary 3.5. It follows from Remark 3.2, p.90 in [34].

Similarly for the problem (3.3-3.4) we have

COROLlary 3.6. Assume that $F_{0} \in H, f, v \in C^{\infty}([0, T] ; H)$. Iffor each $k \in \mathbb{N} v^{(k)}$ satisfies the condition (3.7), then the solution of the problem (3.3-3.4) is in $C^{\infty}((0, T] \times D)$.

ProOF OF COROLlary 3.6. It follows from Remark 3.2, p.90 in [34].

The main result in this section is the following.

THEOREM 3.7. Suppose that $F_{0} \in H, G_{0} \in H$ and $v \in$ $\bigcup_{\delta_{0}>0} L^{2+\frac{6}{\delta_{0}}}\left(0, T ; \mathbb{L}^{3+\delta_{0}}(D)\right)$. Let $F$ and $G$ be solutions of respectively problems (3.11) and (3.12) below.

$$
\begin{aligned}
\frac{\partial F}{\partial t} & =-\nu A F-B(v(t), F), t \in(0, T), \\
F(0, \cdot) & =F_{0}, \\
\frac{\partial G}{\partial t} & =-\nu A G+\operatorname{curl}(v(T-t) \times G), t \in(0, T), \\
G(0, \cdot) & =G_{0} .
\end{aligned}
$$

Then, the following identity holds

$$
(F(t), G(T-t))_{H}=(F(0), G(T))_{H}, t \in[0, T] .
$$


From now on we will only consider the case $D=\mathbb{R}^{3}$. We notice that if $F$ is a solution of the problem (3.1-3.2) with data $\left(F_{0}, f, v\right)$, then $\operatorname{curl} F$ is a solution of the problem (3.3$3.4)$ with data $\left(\operatorname{curl} F_{0}, \operatorname{curl} f, v\right)$.

DEFINITION 3.8. Let $\mathcal{T}_{T}^{v}: H \rightarrow H$ be the vector transport operator defined by $\mathcal{T}_{T}^{v}\left(F_{0}\right)=F(T)$, where $F$ is the unique solution of the problem (3.11) with data $\left(F_{0}, v\right)$.

Define also the time reversal operator

$$
S_{T}: \bigcup_{\delta_{0}>0} L^{2+\frac{6}{\delta_{0}}}\left(0, T ; \mathbb{L}^{3+\delta_{0}}(D)\right) \rightarrow \bigcup_{\delta_{0}>0} L^{2+\frac{6}{\delta_{0}}}\left(0, T ; \mathbb{L}^{3+\delta_{0}}(D)\right)
$$

by $\left(S_{T} v\right)(t)=-v(T-t)$. Then from Theorem 3.7 we infer that

COROLlary 3.9. Assume that $F_{0} \in V, G_{0} \in H$ and $v \in$ $\bigcup_{\delta_{0}>0} L^{2+\frac{6}{\delta_{0}}}\left(0, T ; \mathbb{L}^{3+\delta_{0}}\left(\mathbb{R}^{3}\right)\right)$. Then the following duality relation holds,

$$
\left(\operatorname{curl} F_{0}, \mathcal{T}_{T}^{S_{T}^{v}} G_{0}\right)_{H}=\left(\operatorname{curl} \mathcal{T}_{T}^{v} F_{0}, G_{0}\right)_{H} .
$$

COROLlary 3.10. Assume that $v$ satisfies the assumption (3.5) such that duality relation (3.14) holds. Then

$$
\left\|\mathcal{T}_{T}^{v}\right\|_{\mathcal{L}\left(\mathbb{H}_{h, \mathrm{sol}}^{\alpha, 2}, \mathbb{H}_{h, \mathrm{sol}}^{\alpha, 2}\right)}=\left\|\mathcal{T}_{T}^{S_{T}^{v}}\right\|_{\mathcal{L}\left(\mathbb{H}_{h, \mathrm{sol}}^{1-\alpha, 2}, \mathbb{H}_{h, \mathrm{sol}}^{1-\alpha, 2}\right)}, \alpha \in[0,1]
$$

Proof OF COROLlary 3.10. Because $\mathbb{H}_{h, \text { sol }}^{\alpha, 2}$ is the complex interpolation space between $\mathbb{H}_{h \text {,sol }}^{0,2}=\mathbb{L}_{\text {sol }}^{2}$ and $\mathbb{H}_{h \text {,sol }}^{1,2}$ of order $\alpha$, it is enough to consider the cases $\alpha \in\{0,1\}$. Furthermore, we can restrict ourselves to the case of $\alpha=0$ because $S_{T} \circ S_{T}=\mathrm{id}$.

From equality (3.14) it follows that

$$
\begin{aligned}
& \left\|\mathcal{T}_{T}^{v}\right\|_{\mathcal{L}\left(\mathbb{L}_{\text {sol }}^{2}, \mathbb{L}_{\text {sol }}^{2}\right)}=\sup _{\phi, \psi \in \mathcal{D}\left(\mathbb{R}^{3}\right)} \frac{\left|\left\langle\mathcal{T}_{T}^{v} \phi, \psi\right\rangle\right|}{\|\phi\|_{\mathbb{L}_{\text {sol }}^{2}\|\psi\|_{\mathbb{L}_{\text {sol }}^{2}}}}= \\
& \sup _{\phi, \psi \in \mathcal{D}\left(\mathbb{R}^{3}\right)} \frac{\left|\left\langle\operatorname{curl} \mathcal{T}_{T}^{v} \phi, \operatorname{curl}^{-1} \psi\right\rangle\right|}{\|\phi\|_{\mathbb{L}_{\text {sol }}^{2}}\|\psi\|_{\mathbb{L}_{\text {sol }}^{2}}}= \\
& \sup _{\phi, \psi \in \mathcal{D}\left(\mathbb{R}^{3}\right)} \frac{\left|\left\langle\operatorname{curl} \phi, \mathcal{T}_{T}^{S_{T} v} \operatorname{curl}^{-1} \psi\right\rangle\right|}{\|\phi\|_{\mathbb{L}_{\text {sol }}^{2}}\|\psi\|_{\mathbb{L}_{\text {sol }}^{2}}}= \\
& \sup _{\phi, \psi \in \mathcal{D}\left(\mathbb{R}^{3}\right)} \frac{\left|\left\langle\phi, \mathcal{T}_{T}^{S_{T} v} \psi\right\rangle\right|}{\|\phi\|_{\mathbb{H}_{h, \text { sol }}^{-1,2}}\|\psi\|_{\mathbb{H}_{h, \text { sol }}^{1,2}}}= \\
& \left\|\mathcal{T}_{T}^{S_{T} v}\right\|_{\mathcal{L}\left(\mathbb{H}_{h, \text { sol }}^{1,2}, \mathbb{H}_{h, \text { sol }}^{1,2}\right)}
\end{aligned}
$$

DEFINITION 3.11. By $X_{\alpha}$ we denote the class of all functions $u:[0, \infty) \times \mathbb{R}^{3} \rightarrow \mathbb{R}^{3}$ satisfying the following three conditions.

(i) $u \in L_{l o c}^{\infty}([0, \infty) ; H)$.

(ii) For all $t \in[0, \infty)$ there exists a unique solution of equation (3.11) with parameters $u^{t}=\left.u\right|_{[0, t]}$ and $v^{t}=S^{t}\left(\left.u\right|_{[0, t]}\right)$. Furthermore, the duality relation (3.14) with the vector field $v$ replaced by the vector field $u^{t}$ holds.

(iii) For every $t \in[0, \infty), \mathcal{T}_{t}^{u^{t}} \in \mathcal{L}\left(\mathbb{H}_{h, \text { sol }}^{\alpha, 2}, \mathbb{H}_{h, \text { sol }}^{\alpha, 2}\right)$.

Then the following result follows from Corollary 3.10 
COROllary 3.12. Assume that $\alpha \in[0,1]$. Then $X_{\alpha}=X_{1-\alpha} \subset X_{\frac{1}{2}}$ and the space $X_{\frac{1}{2}}$ is invariant with respect to scalings $\Psi_{\lambda}, \lambda \in(0,1]$, where $\left(\Psi_{\lambda} u\right)(t, x)=\lambda u\left(\lambda^{2} t, \lambda x\right)$, $t \in[0, \infty), x \in \mathbb{R}^{3}$.

Proof of Corollary 3.12. Property $X_{\alpha}=X_{1-\alpha}$ is a direct consequence of Corollary 3.10 and the definition of $X_{\alpha}$. We will show that $X_{\alpha} \subset X_{\frac{1}{2}}$. Let $u \in X_{\alpha}$. Then for all $t \geq 0$,

$$
\mathcal{T}_{t}^{u} \in \mathcal{L}\left(\mathbb{H}_{h, \text { sol }}^{\alpha, 2}, \mathbb{H}_{h, \text { sol }}^{\alpha, 2}\right), \mathcal{T}_{t}^{u} \in \mathcal{L}\left(\mathbb{H}_{h, \text { sol }}^{1-\alpha, 2}, \mathbb{H}_{h, \text { sol }}^{1-\alpha, 2}\right)
$$

Indeed, it follows by definition of $X_{\alpha}$ that

$$
\left|\mathcal{T}_{t}^{u}\right|_{\mathcal{L}\left(\mathbb{H}_{h, \mathrm{sol}}^{1-\alpha, 2}, \mathbb{H}_{h, \mathrm{sol}}^{1-\alpha, 2}\right)}=\left|\mathcal{T}_{t}^{S^{t}\left(\left.u\right|_{[0, t]}\right)}\right|_{\mathcal{L}\left(\mathbb{H}_{h, \mathrm{sol}}^{\alpha, 2}, \mathbb{H}_{h, \mathrm{sol}}^{\alpha, 2}\right)}, t \in[0, \infty)
$$

Therefore, by the Interpolation Theorem, see [35, Theorems 1.9.4, p. 59 and 1.15.3, p. 103], we have that

$$
\mathcal{T}_{t}^{u} \in \mathcal{L}\left(\left[\mathbb{H}_{h, \text { sol }}^{\alpha, 2}, \mathbb{H}_{h, \text { sol }}^{1-\alpha, 2}\right]_{1 / 2},\left[\mathbb{H}_{h, \text { sol }}^{\alpha, 2}, \mathbb{H}_{h, \text { sol }}^{1-\alpha, 2}\right]_{1 / 2}\right), t \in[0, \infty)
$$

i.e.

$$
\mathcal{T}_{t}^{u} \in \mathcal{L}\left(\mathbb{H}_{h, \mathrm{sol}}^{\frac{1}{2}, 2}, \mathbb{H}_{h, \mathrm{sol}}^{\frac{1}{2}, 2}\right), t \in[0, \infty) .
$$

Third property follows from identity

$$
\mathcal{T}_{t}^{\Psi_{\lambda}(u)} \Psi_{\lambda}\left(F_{0}\right)=\Psi_{\lambda}\left(\mathcal{T}_{t}^{u} F_{0}\right), t \in[0, \infty)
$$

and boundedness of scaling operators $\Psi_{\lambda}$ and $\Psi_{\lambda}^{-1}=\Psi_{\frac{1}{\lambda}}$ in $\mathbb{H}_{h, \text { sol }}^{\frac{1}{2}, 2}$.

The first part of our next result is the classical result of Serrin-Prodi- Ladyzhenskaya $([29,27,17])$. But the second part, i.e. inequalities (3.16) and (3.17) are new.

COROLlary 3.13. Assume that $u$ is a weak solution of the NSEs (1.1) with the external force 0. Assume that $u$ satisfies the Serrin condition, i..e $u \in$ $\bigcup_{\delta_{0}>0} L^{2+\frac{6}{\delta_{0}}}\left(0, T ; \mathbb{L}^{3+\delta_{0}}\left(\mathbb{R}^{3}\right)\right)$ and $u(0) \in V$. Then $u \in L^{\infty}(0, T ; V)$, i.e. $u$ is a strong solution of (1.1). Moreover, if $G_{0} \in H$, then

$$
\begin{array}{r}
\left(\operatorname{curl} u(0), \mathcal{T}_{T}^{S_{T}(u)} G_{0}\right)_{H}=\left(\operatorname{curl} u(T), G_{0}\right)_{H}, \\
\|\operatorname{curl} u(T)\|_{H} \leq\left\|\mathcal{T}_{T}^{S_{T}(u)}\right\|_{\mathcal{L}(H, H)}\|\operatorname{curl} u(0)\| \cdot H
\end{array}
$$

REMARK 3.14. Let us observe that the equality (3.16) is a generalization of the helicity invariance $\int_{\mathbb{R}^{3}}(u, \operatorname{curl} u)_{\mathbb{R}^{3}} d x$, see e.g. p. $120-121$ in [24] for the solutions of the Euler equations. Indeed, if we consider the transport operator $\mathcal{T}_{\dot{T}}$ for $\nu=0$ and take $G_{0}=u(T)$ on the right hand side of equality (3.16) then, under the assumption that the Euler equation has a unique solution, we infer that $\mathcal{T}_{T}^{S_{T}(u)} u(T)=u(0)$.

Proof of Corollary 3.13. By Proposition 3.2 there exist unique solution $F \in$ $L^{2}(0, T ; V) \cap L^{\infty}(0, T ; H)$ of equation (3.1-3.2) with initial condition $F_{0}=u(0)$ and $v=u$. We can notice that $u$ is also solution of (3.1-3.2) by Navier-Stokes equation. Thus, $F=u$ and we have (3.16) by Theorem 3.7. Therefore, we have

$$
\|\operatorname{curl} u(t)\|_{H} \leq\left\|\mathcal{T}_{T}^{S_{T}(u)}\right\|_{\mathcal{L}(H, H)}\|\operatorname{curl} u(0)\|_{H}
$$

and by boundedness of operator $\mathcal{T}_{T}^{S_{T}(u)}$ (Proposition 3.2) we get the result. 


\section{Formulae of Feynman-Kac Type.}

The aim of this section is twofold. Firstly, we will discuss the physical meaning of the operator $\mathcal{T}_{T}^{S_{T}(\cdot)}$. Secondly, we will deduce a formula of Feynman-Kac type. In the whole section we suppose that $D=\mathbb{R}^{n}$. We also assume that $\left(\Omega, \mathcal{F},\left\{\mathcal{F}_{t}\right\}_{t \geq 0}, \mathbb{P}\right)$ is a complete filtered probability space and that $(W(t))_{t \geq 0}$ is an $\mathbb{R}^{m}$-valued Wiener process on this space. We have the following Proposition.

Proposition 4.1. Assume that $\alpha \in(0,1), \sigma(\cdot, \cdot) \in L^{1}\left(0, T ; C_{b}^{2, \alpha}\left(\mathbb{R}^{n}, \mathbb{R}^{n} \otimes \mathbb{R}^{m}\right)\right)$, $a(\cdot, \cdot) \in L^{1}\left(0, T ; C_{b}^{1, \alpha}\left(\mathbb{R}^{n}, \mathbb{R}^{n}\right)\right)$. Let us assume that a continuous and adapted process $X=[0, T] \times \mathbb{R}^{n} \times \Omega \rightarrow \mathbb{R}^{n}$ is a unique solution to the problem

$$
\begin{aligned}
& d X_{t}(x)=a\left(t, X_{t}(x)\right) d t+\sigma\left(t, X_{t}(x)\right) d W(t), \\
& X_{0}(x)=x .
\end{aligned}
$$

Then for any $C^{1}$ class closed loop $\Gamma$ in $\mathbb{R}^{n}$, any $F \in C^{1,2}\left([0, T] \times \mathbb{R}^{n}, \mathbb{R}^{n}\right)$ and any $t \in[0, T]$, we have $\mathbb{P}$-a.s.,

$$
\begin{aligned}
& \int_{X_{t}(\Gamma)} \sum_{k=1}^{n} F^{k}(t, x) d x_{k}=\int_{\Gamma} \sum_{k=1}^{n} F^{k}(0, x) d x_{k} \\
& +\int_{0}^{t} \int_{X_{s}(\Gamma)} \sum_{k=1}^{n}\left(\frac{\partial F^{k}}{\partial t}+\sum_{j=1}^{n} a^{j}\left(\frac{\partial F^{k}}{\partial x_{j}}-\frac{\partial F^{j}}{\partial x_{k}}\right)\right. \\
& \left.+\frac{1}{2} \sum_{i, j=1}^{n} \frac{\partial^{2} F^{k}}{\partial x_{i} \partial x_{j}} \sum_{m=1}^{n} \sigma^{i m} \sigma^{j m} b i g g\right) d x_{k} d s \\
& +\frac{1}{2} \int_{0}^{t} \int_{X_{s}(\Gamma)} \sum_{k=1}^{n}\left(\sum_{j, l} \frac{\partial F^{j}}{\partial x_{l}} \sum_{m} \sigma^{l m} \frac{\partial \sigma^{j m}}{\partial x_{k}}\right) d x_{k} d s \\
& +\int_{0}^{t} \int_{X_{s}(\Gamma)} \sum_{k, j=1}^{n} F^{j}(s, x) \frac{\partial \sigma^{j l}}{\partial x_{k}} d x_{k} d W^{l}(s) \\
& +\int_{0}^{t} \int_{X_{s}(\Gamma)} \sum_{k=1}^{n}\left(\sum_{i, l=1} \frac{\partial F^{k}}{\partial x_{i}} \sigma^{i l}\right) d x_{k} d W^{l}(s) .
\end{aligned}
$$

REMARK 4.2. The term (4.2) is of major interest for us. Its appearance allows us to "emulate" drift in two dimensional case i.e. to consider flow without drift such that this term "creates" necessary drift (see subsections 4.1, 4.2 and Theorem 4.12 for detailed explanation).

Proof OF Proposition 4.1. It follows from Theorems 3.3.3, p.94 and 4.6.5, p.173 of [16] that $X_{t}(\cdot), t \in[0, T]$ is a flow of $C^{1}$-diffeomorphisms and $\nabla X_{t}(\cdot)$ satisfies corresponding equation for gradient of the flow. Then formula (4.1) immediately follows from the Itô formula, see [26] for calculations.

COROLlaRy 4.3. Assume that $\nu>0$ and $v \in L^{1}\left(0, T ; C_{b}^{1, \alpha}\left(\mathbb{R}^{n}, \mathbb{R}^{n}\right)\right)$ for some $\alpha \in(0,1)$. Let $\left(X_{s}(t ; x)\right)_{0 \leq s \leq t \leq T}$, be a stochastic flow corresponding to the following 
$S D E$

$$
\begin{aligned}
d X_{s}(t ; x) & =v\left(t, X_{s}(t ; x)\right) d t+\sqrt{2 \nu} d W(t), t \in[s, T], \\
X_{s}(s ; x) & =x .
\end{aligned}
$$

Assume that $F_{0} \in C^{2}\left(\mathbb{R}^{n}\right)$ and let $F \in C^{1,2}\left([0, T] \times \mathbb{R}^{n}, \mathbb{R}^{n}\right)$ be a solution of the following linear equation ${ }^{3}$

$$
\begin{aligned}
\frac{\partial F(t)}{\partial t} & =-\nu A F+\mathrm{P}\left(\left(\nabla F-\nabla F^{\perp}\right) v(T-t)\right), t \in(0, T), \\
F(0) & =F_{0},
\end{aligned}
$$

Then for any $s \in[0, T]$ a process $\left(M_{s}(t)\right)_{t \in[T-s, T]}$ defined by the following formula

$$
M_{s}(t)=\int_{X_{T-s}(t ; \Gamma)} \sum_{k=1}^{n} F^{k}(T-t) d x_{k}, t \in[T-s, T]
$$

is a local martingale.

ProOF OF COROLlary 4.3. This follows immediately from Proposition 4.1.

REMARK 4.4. Corollary 4.3, whose idea is taken from [26], can be seen as a generalization of the Kelvin circulation Theorem, see e.g. [22, p. 26]. Indeed, if $\nu=0$, then $X_{s}(t ; x)$ is a position of a particle at time $t$ starting from point $x$ at time $s$, moving in the deterministic velocity field $v$. Moreover, $M_{s}$ is the circulation along a curve $\gamma$ moved by the flow generated by $v$. Hence, by Proposition 4.1 it follows that the local martingale $M_{s}$ is constant in time. A similar result has recently been independently derived by Constantin and Iyer, see [6, Proposition 2.9].

Next we deduce from the corollary 4.3 the following formula of the Feynman-Kac type for the solution of equation (4.4).

Proposition 4.5. Assume that $v \in L^{1}\left(0, T ; C_{b}^{2, \alpha}\left(\mathbb{R}^{n}, \mathbb{R}^{n}\right)\right)$ for some $\alpha \in(0,1)$ and

$$
\int_{0}^{T}\left(|v|_{L^{\infty}}(s)+|\nabla v|_{L^{\infty}}(s)\right) d s<\infty .
$$

Assume that $F:[0, T] \times \mathbb{R}^{n} \rightarrow \mathbb{R}^{n}$ is a solution of the problem (4.4)-(4.5) with $F_{0} \in$ $C^{2}\left(\mathbb{R}^{n}\right) \cap L^{4}\left(\mathbb{R}^{n}\right)$ and $\left(X_{s}(t ; x)\right)_{0 \leq s \leq t \leq T}$ is a stochastic flow corresponding to SDE (4.3). Assume also that there exists $\beta>0$ such that for any $\Gamma \in C^{1}\left(\mathbb{S}^{1}, \mathbb{R}^{n}\right)$, where $\mathbb{S}^{1}$ is the unit circle, for all $s, t \in[0, T]$ such that $T-s \leq t$,

$$
\mathbb{E}\left|\int_{X_{T-s}(t ; \Gamma)} \sum_{k=1}^{n} F^{k}(T-t, x) d x_{k}\right|^{1+\beta}<\infty .
$$

Fix $s \in[0, T]$ and define a functions $Q_{s}: \mathbb{R}^{n} \rightarrow \mathbb{R}^{n}$ by

$$
\left.Q_{s}(x):=\mathbb{E}\left(F_{0}\left(X_{T-s}(T ; x)\right) \nabla X_{T-s}(T ; x)\right)\right), \quad x \in \mathbb{R}^{n} .
$$

Then, $Q_{s} \in L^{2}\left(\mathbb{R}^{n}, \mathbb{R}^{n}\right) \cap C^{1+\varepsilon}\left(\mathbb{R}^{n}, \mathbb{R}^{n}\right), 0<\varepsilon<\alpha$ and

$$
F(s, x)=\left[\mathrm{P}\left(Q_{s}\right)\right](x), x \in \mathbb{R}^{n}, s \in[0, T] .
$$

\footnotetext{
3 which coincides with Problem (3.11) in the case $n=3$
} 
REMARK 4.6. In connection with the formula (4.8) we can ask whether the flow $\left(X_{s}(t ; x)\right)_{0 \leq s \leq t \leq T}$ associated to the SDE (4.3) is the only flow such that the function $F$ defined by the formula (4.8) is a solution to problem (4.4-4.5)? It turns out that the answer to this question is negative. In the subsections 4.1 and 4.2 we will consider separately two and three dimensional examples.

REMARK 4.7. Condition (4.7) is satisfied if, for instance, $F \in L^{\infty}\left([0, T] \times \mathbb{R}^{n}\right)$ and

$$
\int_{0}^{T}|\nabla v|_{L^{\infty}}(s) d s<\infty .
$$

Indeed, in this case we have the following inequality

$$
\left|\nabla X_{s}(t ; \cdot)\right|_{L^{\infty}} \leq \exp \left(\int_{s}^{t}|\nabla v|_{L^{\infty}}(r) d r\right), s \leq t \leq T,
$$

and hence the result follows.

Proof of Proposition 4.5. For fixed $s \in[0, T)$ let us denote

$$
M_{s}(t)=\int_{X_{T-s}(t ; \Gamma)} \sum_{k=1}^{n} F^{k}(T-t) d x_{k}, t \in[T-s, T] .
$$

Then by Corollary 4.3 the process $\left(M_{s}(t)\right), t \in[T-s, T]$ is a local martingale. Hence, by the uniform integrability condition (4.7) we infer that $M_{s}$ is martingale and so $\mathbb{E} M_{s}(T-$ $s)=\mathbb{E} M_{s}(T)$. In particular,

$$
\int_{\Gamma} F^{k}(s, x) d x_{k}=\int_{\Gamma} Q_{s}^{k}(x) d x_{k}, \Gamma \in C^{1}\left(S^{1}, \mathbb{R}^{n}\right) .
$$

It immediately follows from Theorems 3.3.3, p.94 and 4.6.5, p.173 of [16] that $Q_{s} \in$ $C^{1+\varepsilon}\left(\mathbb{R}^{n}, \mathbb{R}^{n}\right), 0<\varepsilon<\alpha$. Furthermore, $Q_{s} \in L^{2}\left(\mathbb{R}^{n}, \mathbb{R}^{n}\right)$. Indeed, by the definition of the flow (4.3) we have

$$
\sup _{x}\left|\nabla X_{T-s}(T ; x)\right| \leq e^{\int^{T}|\nabla v|_{L} \infty(r) d r} .
$$

Hence

$$
\begin{aligned}
\int_{\mathbb{R}^{n}}\left|Q_{s}(x)\right|^{2} d x & \leq \int_{\mathbb{R}^{n}} \mathbb{E}\left|F_{0}\left(X_{T-s}(T ; x)\right) \nabla X_{T-s}(T ; x)\right|^{2} d x \\
& \leq \mathbb{E}\left(\sup _{x}\left|\nabla X_{T-s}(T ; x)\right|^{2} \int_{\mathbb{R}^{n}}\left|F_{0}\left(X_{T-s}(T ; x)\right)\right|^{2} d x\right) \\
& \leq e^{\int^{T}|\nabla v|_{L^{\infty}}(r) d r} \mathbb{E} \int_{\mathbb{R}^{n}}\left|F_{0}\left(X_{T-s}(T ; x)\right)\right|^{2} d x \\
& \leq \int^{\int^{T}|\nabla v|_{L}(r) d r} \int_{\mathbb{R}^{n}} \tilde{\mathbb{E}}\left(\left|F_{0}\left(x+\sqrt{2 \nu}\left(W_{T}-W_{T-s}\right)\right)\right|^{2} \mathcal{E}_{T-s}^{T}\right) d x,
\end{aligned}
$$


where

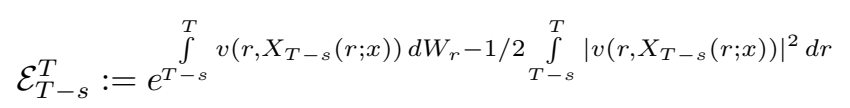

is a stochastic exponent. We can notice that

$$
\tilde{\mathbb{E}}\left|\mathcal{E}_{T-s}^{T}\right|^{2} \leq e^{2 \int_{0}^{T}|v(r)|_{L} \infty(r) d r}
$$

and, therefore, combining (4.11) and (4.12) we get

$$
\int_{\mathbb{R}^{n}}\left|Q_{s}(x)\right|^{2} d x \leq e^{\int^{T}\left(|v|_{L} \infty(r)+|\nabla v|_{L} \infty(r)\right) d r} \int_{\mathbb{R}^{n}}\left|F_{0}\right|^{4} d x<\infty .
$$

It remains to notice that operator $\mathrm{P}: C^{\beta}\left(\mathbb{R}^{n}, \mathbb{R}^{n}\right) \rightarrow C^{\beta}\left(\mathbb{R}^{n}, \mathbb{R}^{n}\right), \beta \in(0,1)$ is bounded. Indeed, it follows from representation of $\mathrm{P}$ as pseudodifferential operator ([11],[33]) and Theorem 7.9.6 in [12].

REMARK 4.8. Another method of proving the formula (4.8) is presented in the article [5] by Busnello et al., see also literature therein. The approach used there is based upon an extension of the standard Feynman-Kac formula for parabolic equations to more general system of linear parabolic equations with a potential term (see the system (3.2) in [5, p.306]). This extension is carried out by using the new variables method introduced earlier by Krylov [15]. One should mention here that the formula (4.8) is used in [5] to prove the local existence and uniqueness of strong solutions to the NSEs.

4.1. Examples of nontrivial flows in $\mathbb{R}^{2}$. In this subsection we provide nontrivial examples of the flows which can be used in the Feynman-Kac type formula (4.8) in two dimensional case.

Proposition 4.9. Suppose that $v \in C_{0}^{\infty}\left([0, T] \times \mathbb{R}^{2}, \mathbb{R}^{2}\right), \psi: \mathbb{R} \rightarrow \mathbb{R}$ is a $C^{1}$-class diffeomorphism, $\phi=\psi \circ \operatorname{rot} v$ and $F_{0} \in C_{0}^{\infty}\left(\mathbb{R}^{n}\right)$. Let $\left(X_{s}(t ; x)\right), 0 \leq s \leq t \leq T$ be the stochastic flow corresponding to the following $S D E$

$$
\begin{aligned}
d X_{s}(t ; x) & =v\left(t, X_{s}(t ; x)\right) d t+\sqrt{2 \nu} \sigma_{1}\left(X_{s}(t ; x)\right) d W(t), \\
X_{s}(s ; x) & =x,
\end{aligned}
$$

where

$$
\sigma_{1}(x)=\left(\begin{array}{cc}
\cos \phi(x) & -\sin \phi(x) \\
\sin \phi(x) & \cos \phi(x)
\end{array}\right), x \in \mathbb{R}^{2} .
$$

Assume that $F:[0, T] \times \mathbb{R}^{n} \rightarrow \mathbb{R}^{n}$ is a solution to problem (4.4-4.5) such that for some $\beta>0$ and any $\Gamma \in C^{1}\left(\mathbb{S}^{1}, \mathbb{R}^{2}\right)$ the condition (4.7) is satisfied. Then, the formula (4.8) holds true.

Proof of Proposition 4.9. Suppose that the condition (4.7) is fulfilled. Then, it is enough to show that process $\left(M_{s}(t)\right), t \in[T-s, T]$ defined by formula (4.9) above (where flow $\left(X_{s}(t ; x)\right), 0 \leq s \leq t \leq T$ is given by (4.14)) is a local martingale. We have

$$
\int_{X_{T-s}(t ; \Gamma)} \sum_{k=1}^{n} F^{k}(T-t, x) d x_{k}=\int_{\Gamma} \sum_{k=1}^{n} F^{k}(s, x) d x_{k}
$$




$$
\begin{aligned}
& +\int_{T-s X_{T-s}(\tau ; \Gamma)}^{t} \sum_{k=1}^{n}\left(\frac{\partial F^{k}}{\partial t}+\sum_{j=1}^{n} v^{j}\left(\frac{\partial F^{k}}{\partial x_{j}}-\frac{\partial F^{j}}{\partial x_{k}}\right)\right. \\
& \left.+\nu \sum_{i, j=1}^{n} \frac{\partial^{2} F^{k}}{\partial x_{i} \partial x_{j}} \sum_{m=1}^{n} \sigma_{1}^{i m} \sigma_{1}^{j m}\right) d x_{k} d \tau+ \\
& +\nu \int_{T-s}^{t} \int_{X_{T-s}(\tau ; \Gamma)} \sum_{k=1}^{n}\left(\sum_{j, l} \frac{\partial F^{j}}{\partial x_{l}} \sum_{m} \sigma_{1}^{l m} \frac{\partial \sigma_{1}^{j m}}{\partial x_{k}}\right) d x_{k} d \tau \\
& +\sqrt{2 \nu} \int_{T-s}^{t} \int_{X_{T-s}(\tau ; \Gamma)}^{n} \sum_{k, j=1}^{n} F^{j}(T-\tau, x) \frac{\partial \sigma_{1}^{j l}}{\partial x_{k}} d x_{k} d w_{\tau}^{l} \\
& +\sqrt{2 \nu} \int_{T-s}^{t} \int_{X_{T-s}(\tau ; \Gamma)}^{n}\left(\sum_{k=1}^{n}\left(\sum_{i, l=1} \frac{\partial F^{k}}{\partial x_{i}} \sigma_{1}^{i l}\right) d x_{k} d W_{\tau}^{l} .\right.
\end{aligned}
$$

Hence, because $\sigma_{1}$ is orthogonal matrix and $F$ satisfies (4.4) we have that

$$
\begin{aligned}
\frac{\partial F^{k}}{\partial t} & +\sum_{j=1}^{n} v^{j}\left(\frac{\partial F^{k}}{\partial x_{j}}-\frac{\partial F^{j}}{\partial x_{k}}\right)+\nu \sum_{i, j=1}^{n} \frac{\partial^{2} F^{k}}{\partial x_{i} \partial x_{j}} \sum_{m=1}^{n} \sigma_{1}^{i m} \sigma_{1}^{j m} \\
& =\frac{\partial F^{k}}{\partial t}+\sum_{j=1}^{n} v^{j}\left(\frac{\partial F^{k}}{\partial x_{j}}-\frac{\partial F^{j}}{\partial x_{k}}\right)+\nu \triangle F^{k}=\frac{\partial p}{\partial x_{k}} .
\end{aligned}
$$

Therefore, it is enough to show that

$$
\int_{T-s}^{t} \int_{X_{T-s}(\tau ; \Gamma)} \sum_{k=1}^{n}\left(\sum_{j, l} \frac{\partial F^{j}}{\partial x_{l}} \sum_{m} \sigma_{1}^{l m} \frac{\partial \sigma_{1}^{j m}}{\partial x_{k}}\right) d x_{k} d \tau=0 .
$$

We have that $\sum_{m} \sigma_{1}^{l m} \frac{\partial \sigma_{1}^{j m}}{\partial x_{k}}$ is antisymmetric w.r.t. indexes $l, j$ because $\sigma_{1}$ is orthogonal. Hence $n=2$, it means that it is enough to calculate

$$
\sum_{m} \sigma_{1}^{1 m} \frac{\partial \sigma_{1}^{2 m}}{\partial x_{k}}=\cos \phi \frac{\partial}{\partial x_{k}}(\sin \phi)-\sin \phi \frac{\partial}{\partial x_{k}}(\cos \phi)=\frac{\partial \phi}{\partial x_{k}}
$$

and, therefore,

$$
\begin{aligned}
\int_{T-s}^{t} & \int_{X_{T-s}(\tau ; \Gamma)} \sum_{k=1}^{n}\left(\sum_{j, l} \frac{\partial F^{j}}{\partial x_{l}} \sum_{m} \sigma_{1}^{l m} \frac{\partial \sigma_{1}^{j m}}{\partial x_{k}}\right) d x_{k} d \tau \\
= & \int_{T-s}^{t} \int_{X_{T-s}(\tau ; \Gamma)}\left(\frac{\partial F^{1}}{\partial x_{2}}-\frac{\partial F^{2}}{\partial x_{1}}\right) d \phi d \tau=\int_{T-s}^{t} \int_{X_{T-s}(\tau ; \Gamma)} \psi^{-1}(\phi) d \phi d \tau=0 .
\end{aligned}
$$

REMARK 4.10. The construction of the example from Proposition 4.9 can easily be generalized to the case $n=3$ in the following way. Let $\psi: \mathbb{R} \rightarrow \mathbb{R}$ be a $C^{1}$-class 
diffeomorphism. Define $\phi=\psi \circ(\operatorname{curl} v)^{1}$ and

$$
\sigma_{1}(x)=\left(\begin{array}{ccc}
\cos \phi(x) & -\sin \phi(x) & 0 \\
\sin \phi(x) & \cos \phi(x) & 0 \\
0 & 0 & 1
\end{array}\right), x \in \mathbb{R}^{3}
$$

Let $\left(X_{s}(t ; x)\right)_{0 \leq s \leq t \leq T}$ be a stochastic flow corresponding to the following SDE

$$
\begin{aligned}
(4.15) d X_{s}(t ; x) & =v\left(t, X_{s}(t ; x)\right) d t+\sqrt{2 \nu} \sigma_{1}\left(X_{s}(t ; x)\right) d W(t), 0 \leq s \leq t \leq T \\
X_{s}(s ; x) & =x
\end{aligned}
$$

Then the assertion of Proposition (4.9) holds true.

Note that similar construction can be made for other components of the curl $v$ ) but the truly three dimensional rotations $\sigma_{1}$ will be considered in next paragraph.

REMARK 4.11. Let us note that the laws of the solutions to SDEs (4.14) and (4.3) are the same. Indeed, it is easy to see that quadratic variations of both processes are the same. In the next example we will show that it is possible to find a flow such that its one-point motion has a law of Brownian motion.

THEOREM 4.12. Suppose that $\nu>0, \delta>0$ and a divergence free vector field $v$ : $\mathbb{R}^{2} \rightarrow \mathbb{R}^{2}$ is of $C^{1+\delta}$ class. Let $\phi: \mathbb{R}^{2} \rightarrow \mathbb{R}$ be such that $t^{4} v=\nabla^{\perp} \phi$. Define

$$
\sigma_{1}(x)=\left(\begin{array}{cc}
\cos \frac{\phi(x)}{\nu} & -\sin \frac{\phi(x)}{\nu} \\
\sin \frac{\phi(x)}{\nu} & \cos \frac{\phi(x)}{\nu}
\end{array}\right), x \in \mathbb{R}^{2},
$$

Let us denote by $X_{s}(t ; x), 0 \leq s \leq t \leq T, x \in \mathbb{R}^{2}$ the stochastic flow of diffeomorphisms of $\mathbb{R}^{2}$ of class $C^{2}$ corresponding to the following $S D E$

$$
\left\{\begin{aligned}
d X_{s}(t ; x) & =\sqrt{2 \nu} \sigma_{1}\left(X_{s}(t ; x)\right) d W(t), 0 \leq s \leq t \leq T \\
X_{s}(s ; x) & =x
\end{aligned}\right.
$$

Assume also that $F_{0} \in C^{2}\left(\mathbb{R}^{2}\right) \cap L^{2}\left(\mathbb{R}^{2}\right)$ and that $F:[0, T] \times \mathbb{R}^{2} \rightarrow \mathbb{R}^{2}$ is a solution to problem (4.4-4.5) such that for some $\beta>0$ and any $\Gamma \in C^{1}\left(\mathbb{S}^{1}, \mathbb{R}^{2}\right)$ the condition (4.7) is satisfied. Denote $Q_{s}(x)=\mathbb{E}\left(F_{0}\left(X_{T-s}(T ; x)\right) \nabla X_{T-s}(T ; x)\right)$. Then $Q_{s} \in L^{2}\left(\mathbb{R}^{n}\right) \cap C^{1+\varepsilon}\left(\mathbb{R}^{n}\right), 0<\varepsilon<\delta$ and

$$
F(s, x)=\mathrm{P}\left(Q_{s}\right)(x), s \in[0, T], x \in \mathbb{R}^{n} .
$$

REMARK 4.13. As we have already noticed above the formula (4.17) can be viewed as generalization of Kelvin Theorem as in the Corollary 4.3. Indeed, it is enough to integrate both sides of (4.17) w.r.t. arbitrary smooth closed contour $\Gamma$.

Proof of Theorem 4.12. From Theorem 4.6.5, p. 173 in [16] we infer that there exists a flow $X_{s}(t ; x), 0 \leq s \leq t \leq T$ for problem (4.16) consisting of diffeomorphisms of class $C^{2+\varepsilon}$.

Moreover, it follows from Theorems 3.3.3, p. 94 and 4.6.5, p. 173 therein that for all $s \in[0, T], Q_{s} \in C^{1+\varepsilon}\left(\mathbb{R}^{n}, \mathbb{R}^{n}\right), 0<\varepsilon<\delta$. Let us fix $s \in[0, T]$. We will show now that $Q_{s} \in L^{2}\left(\mathbb{R}^{n}, \mathbb{R}^{n}\right)$. Since by Corollary 4.6 .7 p. 175 of [16] there exists a positive constant $C$ such that

$$
\sup _{x \in \mathbb{R}^{n}} \mathbb{E}\left|\nabla X_{T-s}(T ; x)\right|^{2} \leq C
$$

\footnotetext{
${ }^{4}$ Such $\phi$ exists because $\operatorname{div} v=0$.
} 
by the Hölder inequality we infer that

$$
\begin{array}{r}
\int_{\mathbb{R}^{n}}\left|Q_{s}(x)\right|^{2} d x \leq \int_{\mathbb{R}^{n}} \mathbb{E}\left|F_{0}\left(X_{T-s}(T ; x)\right)\right|^{2} \mathbb{E}\left|\nabla X_{T-s}(T ; x)\right|^{2} d x \\
\leq C \int_{\mathbb{R}^{n}} \mathbb{E}\left|F_{0}\left(X_{T-s}(T ; x)\right)\right|^{2} d x .
\end{array}
$$

Furthermore, let us observe that the law of the one-point motion of the flow $X_{T-s}(T ; x)$ is equal to the law of the Brownian Motion (see example 6.1, p. 75 of [13] for more details). Therefore, we infer that

$$
\int_{\mathbb{R}^{n}} \mathbb{E}\left|F_{0}\left(X_{T-s}(T ; x)\right)\right|^{2} d x=\int_{\mathbb{R}^{n}}\left|S_{s}^{\nu} F_{0}(x)\right|^{2} d x \leq \int_{\mathbb{R}^{n}}\left|F_{0}(x)\right|^{2} d x,
$$

where $\left\{S_{t}^{\nu}=e^{\nu t \triangle}\right\}_{t \geq 0}$ is a heat semigroup. Combining inequalities (4.18) and (4.19) we get

$$
\int_{\mathbb{R}^{n}}\left|Q_{s}(x)\right|^{2} d x \leq C \int_{\mathbb{R}^{n}}\left|F_{0}(x)\right|^{2} d x
$$

Similarly to Proposition 4.9 we get that $\int_{X_{T-s}(t ; \Gamma)} \sum_{k=1}^{3} F^{k}(T-t) d x_{k}, t \in[T-s, T]$ is a local martingale. Indeed, correction term in (4.1) due to rotation of Brownian Motion is equal to $\int_{T-s}^{t} \int_{X_{T-s}(\tau ; \Gamma)}\left(\frac{\partial F^{1}}{\partial x_{2}}-\frac{\partial F^{2}}{\partial x_{1}}\right) d \phi d s$, see the previous Proposition, and if $v=\nabla^{\perp} \phi$ this is exactly first order term of two dimensional equation (4.4).

COROllary 4.14. Let $\left(X_{s}(t ; x)\right) 0 \leq s \leq t \leq T, x \in \mathbb{R}^{2}$ be the stochastic flow corresponding to SDE (4.16). Then

$$
\begin{aligned}
& d\left(\begin{array}{cc}
\frac{\partial X_{s}^{1}(t ; x)}{\partial x_{1}} & \frac{\partial X_{s}^{1}(t ; x)}{\partial x_{2}} \\
\frac{\partial X_{s}^{2}(t ; x)}{\partial x_{1}} & \frac{\partial X_{s}^{2}(t ; x)}{\partial x_{2}}
\end{array}\right) \\
= & \frac{1}{\nu}\left(\begin{array}{cc}
-v_{2}\left(t, X_{s}(t ; x)\right) d X_{s}^{2}(t ; x) & v_{1}\left(t, X_{s}(t ; x)\right) d X_{s}^{2}(t ; x) \\
v_{2}\left(t, X_{s}(t ; x)\right) d X_{s}^{1}(t ; x) & -v_{1}\left(t, X_{s}(t ; x)\right) d X_{s}^{1}(t ; x)
\end{array}\right) \\
& \left(\begin{array}{cc}
\frac{\partial X_{s}^{1}(t ; x)}{\partial x_{1}} & \frac{\partial X_{s}^{1}(t ; x)}{\partial x_{2}} \\
\frac{\partial X_{s}^{2}(t ; x)}{\partial x_{1}} & \frac{\partial X_{s}^{2}(t ; x)}{\partial x_{2}}
\end{array}\right),
\end{aligned}
$$

and

$$
\left(\begin{array}{cc}
\frac{\partial X_{s}^{1}(s ; x)}{\partial x_{1}} & \frac{\partial X_{s}^{1}(s ; x)}{\partial x_{2}} \\
\frac{\partial X_{s}^{2}(s ; x)}{\partial x_{1}} & \frac{\partial X_{s}^{2}(s ; x)}{\partial x_{2}}
\end{array}\right)=\left(\begin{array}{cc}
1 & 0 \\
0 & 1
\end{array}\right) .
$$

Proof of Corollary 4.14. We have by definition of the flow $\left(X_{s}(t ; x)\right), 0 \leq s \leq$ $t \leq T$ that

$$
\begin{gathered}
d X_{s}^{1}(t ; x)=\sqrt{2 \nu}\left(\cos \frac{\phi}{\nu}\left(X_{s}(t ; x)\right) d w_{t}^{1}-\sin \frac{\phi}{\nu}\left(X_{s}(t ; x)\right) d w_{t}^{2}\right) \\
d X_{s}^{1}(t ; x)=\sqrt{2 \nu}\left(\sin \frac{\phi}{\nu}\left(X_{s}(t ; x)\right) d w_{t}^{1}+\cos \frac{\phi}{\nu}\left(X_{s}(t ; x)\right) d w_{t}^{2}\right) \\
X_{s}(s ; x)=x, x \in \mathbb{R}^{2} .
\end{gathered}
$$


Taking derivative of the flow $\left(X_{s}(t ; x)\right), 0 \leq s \leq t \leq T$ with respect to initial condition $x$ we get for the first component of the flow

$$
\begin{gathered}
d\left(\begin{array}{c}
\frac{\partial X_{s}^{1}(t ; x)}{\partial x_{1}} \\
\frac{\partial X_{s}^{1}(t ; x)}{\partial x_{2}}
\end{array}\right)=\sqrt{2 \nu} \\
\left(\begin{array}{c}
\left(-\frac{1}{\nu} \sin \frac{\phi}{\nu}\left(X_{s}(t ; x)\right) d w_{t}^{1}-\frac{1}{\nu} \cos \frac{\phi}{\nu}\left(X_{s}(t ; x)\right) d w_{t}^{2}\right)\left(\frac{\partial \phi}{\partial x_{1}} \frac{\partial X_{s}^{1}(t ; x)}{\partial x_{1}}+\frac{\partial \phi}{\partial x_{2}} \frac{\partial X_{s}^{2}(t ; x)}{\partial x_{1}}\right) \\
\left(-\frac{1}{\nu} \sin \frac{\phi}{\nu}\left(X_{s}(t ; x)\right) d w_{t}^{1}-\frac{1}{\nu} \cos \frac{\phi}{\nu}\left(X_{s}(t ; x)\right) d w_{t}^{2}\right)\left(\frac{\partial \phi}{\partial x_{1}} \frac{\partial X_{s}^{1}(t ; x)}{\partial x_{2}}+\frac{\partial \phi}{\partial x_{2}} \frac{\partial X_{s}^{2}(t ; x)}{\partial x_{2}}\right)
\end{array}\right) \\
=\left(\begin{array}{c}
-\frac{1}{\nu} d X_{s}^{2}(t ; x)\left(v_{2} \frac{\partial X_{s}^{1}(t ; x)}{\partial x_{1}}-v_{1} \frac{\partial X_{s}^{2}(t ; x)}{\partial x_{1}}\right) \\
-\frac{1}{\nu} d X_{s}^{2}(t ; x)\left(v_{2} \frac{\partial X_{s}^{1}(t ; x)}{\partial x_{2}}-v_{1} \frac{\partial X_{s}^{2}(t ; x)}{\partial x_{2}}\right)
\end{array}\right),
\end{gathered}
$$

where in the last inequality we have used that $v=\nabla^{\perp} \phi$ and definition of the flow. Similarly we can get an equation for the gradient of the second component of the flow. The result follows.

Proposition 4.15. Suppose that the vector field $v: \mathbb{R}^{2} \rightarrow \mathbb{R}^{2}$ is of $C_{0}^{\infty}$ class and divergence free, i.e. $\operatorname{div} v=0$. Let $X_{s}(t ; x), 0 \leq s \leq t \leq T$ be the flow corresponding to equation (4.16). Identifying $\mathbb{C}$ with $\mathbb{R}^{2}$ in the usual way, i.e. $z=x_{1}+\imath x_{2}, x=\left(x_{1}, x_{2}\right)$, we can define a flow $Z_{s}(t ; z), 0 \leq s \leq t \leq T, z \in \mathbb{C}$ by $Z_{s}(t ; z)=X_{s}^{1}(t ; x)+\imath X_{s}^{1}(t ; x)$. If $F_{0} \in C_{0}^{\infty}\left(\mathbb{R}^{2}\right)$ and $F:[0, T] \times \mathbb{R}^{2} \rightarrow \mathbb{R}^{2}$ is a solution of equation (4.4) such that for some $\beta>0$ and any smooth closed loop $\Gamma$ condition (4.7) is satisfied, then

$$
\left.\mathbf{F}(t, z)=\mathrm{P}\left[\mathbb{E}\left(\overline{\mathbf{F}_{0}}\left(Z_{T-t}(T ; z)\right)\right) \frac{\partial Z_{T-t}(T ; z)}{\partial \bar{z}}+\mathbf{F}_{0}\left(Z_{T-t}(T ; z)\right)\right) \frac{\partial \overline{Z_{T-t}(T ; z)}}{\partial \bar{z}}\right],
$$

where $\mathbf{F}(t, z)=F^{1}(t, x)+\imath F^{2}(t, x)$ and $\mathbf{v}(t, z)=v^{1}(t, x)+\imath v^{2}(t, x)$.

Moreover, $\frac{\partial Z_{s}(t ; z)}{\partial \bar{z}}, \frac{\partial \overline{Z_{s}(t ; z)}}{\partial \bar{z}}$ satisfy the following system of equations:

$$
\begin{aligned}
d\left(\frac{\partial Z_{s}(t ; z)}{\partial \bar{z}}\right)= & \frac{1}{2 \nu}\left(\mathbf{v}\left(t, Z_{s}(t ; z)\right) \frac{\partial \overline{Z_{s}(t ; z)}}{\partial \bar{z}}-\overline{\mathbf{v}}\left(t, Z_{s}(t ; z)\right) \frac{\partial Z_{s}(t ; z)}{\partial \bar{z}}\right) d Z_{s}(t ; z) \\
d\left(\frac{\partial \overline{Z_{s}(t ; z)}}{\partial \bar{z}}\right)= & \frac{1}{2 \nu}\left(\overline{\mathbf{v}}\left(t, Z_{s}(t ; z)\right) \frac{\partial Z_{s}(t ; z)}{\partial \bar{z}}-\mathbf{v}\left(t, Z_{s}(t ; z)\right) \frac{\partial \overline{Z_{s}(t ; z)}}{\partial \bar{z}}\right) d \overline{Z_{s}(t ; z)} \\
& \frac{\partial Z_{s}(s ; z)}{\partial \bar{z}}=0, \frac{\partial \overline{Z_{s}(s ; z)}}{\partial \bar{z}}=1,
\end{aligned}
$$

where $\overline{-}$ is a complex conjugation.

Proof OF Proposition 4.15. Definition of the flow (4.16) can be reformulated as follows

$$
\left\{\begin{aligned}
d Z_{s}(t ; z)(z, \bar{z}) & =\sqrt{2 \nu} e^{\frac{\imath \phi\left(Z_{s}(t ; z), \overline{Z_{s}(t ; z)}\right)}{\nu}} d W(t)^{\mathbb{C}}, \\
Z_{s}(s ; z) & =z,
\end{aligned}\right.
$$

where $W(t)^{\mathbb{C}}=W(t)^{1}+\imath W(t)^{2}$ - Wiener process in $\mathbb{C}$. Then equation (4.23) immediately follow from definition (4.24). Formula (4.22) is simply rewriting of formula (4.8).

REMARK 4.16. Theorem 4.12 indicates the difference between the passive scalar advection equation and the vector advection equation. In the former case the Feynman-Kac type formula does not contain a gradient of the flow and hence the solution is completely determined by the law of flow itself. Since the rotation of the Brownian Motion does not change the law of the flow, we cannot employ the same trick for the scalar advection equation as we did for the vector advection equation. 
QUESTION 4.17. In connection with Theorem 4.12 we can ask if it is possible to give a direct proof (not through formula (4.1)) of the fact that the limit as $\nu \rightarrow 0$ exists and the limit is a solution to the $2 \mathrm{D}$ Euler equations?

4.2. Examples of nontrivial flows in $\mathbb{R}^{3}$. In this subsection we provide nontrivial examples of the flows which can be used in the Feynman-Kac type formula (4.8) in three dimensional case.

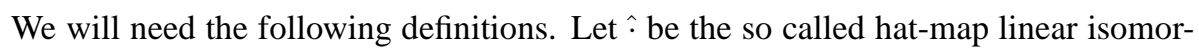
phism defined by

$$
\hat{\imath}: \mathbb{R}^{3} \ni\left(\begin{array}{c}
x_{1} \\
x_{2} \\
x_{3}
\end{array}\right) \mapsto\left(\begin{array}{ccc}
0 & -x_{3} & x_{2} \\
x_{3} & 0 & -x_{1} \\
-x_{2} & x_{1} & 0
\end{array}\right) \in \mathfrak{s o}(3),
$$

where $\mathfrak{s o}(3)$ is the Lie algebra of antisymmetric matrices. Let also $S O(3)$ be the Lie group of orthogonal matrices with determinant equal to one and let $\exp : \mathfrak{s o}(3) \ni A \mapsto e^{A} \in$ $S O(3)$ be the standard exponential map. Let us notice that this map is a surjection.

Denote $S=\operatorname{ker}(\exp )$. Define a map $B C H: \mathfrak{s o}(3) \times \mathfrak{s o}(3) \rightarrow \mathfrak{s o}(3) / S$ by

$$
\exp (B C H(\hat{u}, \hat{v}))=\exp (\hat{u}) \exp (\hat{v}), \hat{u}, \hat{v} \in \mathfrak{s o}(3) .
$$

Now we will find different form of the term (4.2) appearing in formula (4.1) due to diffusion coefficient $\sigma$ of the flow $X$..

Proposition 4.18. Let $a \in C^{1}\left([0, T] \times \mathbb{R}^{3}, \mathbb{R}^{3}\right)$ and a map $\sigma$ is defined by $\sigma$ : $[0, T] \times \mathbb{R}^{3} \ni(t, x) \mapsto \exp (\widehat{a(t, x)}) \in S O(3)$. If $|a|(t, x) \neq 0$, then

$$
\sum_{m} \sigma^{\cdot m} \frac{\partial \sigma^{\cdot m}}{\partial x_{k}}=(1-\cos |a|) b \widehat{\times \frac{\partial b}{\partial x_{k}}}+\sin |a| \widehat{\frac{\partial b}{\partial x_{k}}}+\widehat{b} \frac{\partial|a|}{\partial x_{k}}
$$

where $\vec{b}=\frac{\vec{a}}{|a|}$. If $|a|(t, x)=0$ then

$$
\sum_{m} \sigma^{\cdot m} \frac{\partial \sigma^{\cdot m}}{\partial x_{k}}=\frac{\partial \vec{a}}{\partial x_{k}}
$$

REMARK 4.19. We can notice that the right side if equality (4.25) can be rewritten as follows

$$
(1-\cos |a|) b \widehat{\times \frac{\partial b}{\partial x_{k}}}+(\sin |a|-|a|) \frac{\widehat{\partial b}}{\partial x_{k}}+\frac{\partial \vec{a}}{\partial x_{k}} .
$$

Therefore it converges to $\frac{\partial \vec{a}}{\partial x_{k}}$ when $|a| \rightarrow 0,|a| \neq 0$. Hence, in the following considerations we will not to single out the case of $|a|(t, x)=0$.

Proof of Proposition 4.18. If $a(t, x)=0$ then formula (4.26) immediately follows from definition of $\sigma$. Assume that $a(t, x) \neq 0$. We will use the following BakerCampbell-Hausdorff formula in $\mathfrak{s o}(3)$, see e.g. [8, p. 630].

Proposition 4.20. If $u, v \in \mathbb{R}^{3}$ then

$$
B C H(\hat{u}, \hat{v})=\alpha \hat{u}+\beta \hat{v}+\gamma[\hat{u}, \hat{v}]
$$

where $[\hat{u}, \hat{v}]$ denotes the commutator of $\hat{u}$ and $\hat{v}$, and $\alpha, \beta$, and $\gamma$ are real constants defined by

$$
\alpha=\frac{\sin ^{-1}(d)}{d} \frac{a_{1}}{\theta}, \beta=\frac{\sin ^{-1}(d)}{d} \frac{b_{1}}{\phi}, \gamma=\frac{\sin ^{-1}(d)}{d} \frac{c_{1}}{\theta \phi}
$$


where $a_{1}, b_{1}, c_{1}$ and $d$ are defined as

$$
\begin{aligned}
a_{1} & =\sin \theta \cos ^{2}(\phi / 2)-\sin \phi \sin ^{2}(\theta / 2) \cos \angle(u, v), \\
b_{1} & =\sin \phi \cos ^{2}(\theta / 2)-\sin \theta \sin ^{2}(\phi / 2) \cos \angle(u, v), \\
c_{1} & =\frac{1}{2} \sin (\theta) \sin (\phi)-2 \sin ^{2}(\theta / 2) \sin ^{2}(\phi / 2) \cos \angle(u, v), \\
d & =\sqrt{a_{1}^{2}+b_{1}^{2}+2 a_{1} b_{1} \cos \angle(u, v)+c_{1}^{2} \sin ^{2} \angle(u, v)} .
\end{aligned}
$$

In the above formulae $\theta=|u|, \phi=|v|$, and $\angle(u, v)$ is the angle between the two vectors $u$ and $v$.

We have

$$
\begin{aligned}
& \sum_{m} \sigma^{\cdot m} \frac{\partial \sigma^{\cdot m}}{\partial x_{k}} \\
= & \exp (-\hat{a}) \frac{\partial}{\partial x_{k}} \exp (\hat{a})=\exp (-\hat{a}) \times \lim _{\delta \rightarrow 0} \frac{1}{\delta}\left(\exp \left(\hat{a}\left(x+\delta e_{k}\right)\right)-\exp (\hat{a}(x))\right) \\
= & \lim _{\delta \rightarrow 0} \frac{1}{\delta}\left(\exp (-\hat{a}) \exp \left(\hat{a}\left(x+\delta e_{k}\right)\right)-\mathrm{id}\right) \\
= & \lim _{\delta \rightarrow 0} \frac{1}{\delta}\left(\exp \left(B C H\left(-\hat{a}, \hat{a}\left(x+\delta e_{k}\right)\right)\right)-\mathrm{id}\right)=\lim _{\delta \rightarrow 0} \frac{B C H\left(-\hat{a}, \hat{a}\left(x+\delta e_{k}\right)\right)}{\delta} \\
= & \lim _{\delta \rightarrow 0} \frac{\alpha(\delta)(-\hat{a}(x))+\beta(\delta) \hat{a}\left(x+\delta e_{k}\right)+\gamma(\delta)\left[-\hat{a}(x), \hat{a}\left(x+\delta e_{k}\right)\right]}{\delta}=(*),
\end{aligned}
$$

where in the last equality we have used Proposition 4.18 with $u=-\hat{a}(x), v=\hat{a}\left(x+\delta e_{k}\right)$. Therefore,

$$
\begin{aligned}
&(*)= \lim _{\delta \rightarrow 0} \beta(\delta) \frac{\hat{a}\left(x+\delta e_{k}\right)-\hat{a}}{\delta}+\hat{a}(x) \lim _{\delta \rightarrow 0} \frac{\beta(\delta)-\alpha(\delta)}{\delta} \\
&-\lim _{\delta \rightarrow 0} \gamma(\delta)\left[\hat{a}(x), \frac{\hat{a}\left(x+\delta e_{k}\right)-\hat{a}(x)}{\delta}\right] \\
&=\frac{\partial \hat{a}}{\partial x_{k}} \lim _{\delta \rightarrow 0} \beta(\delta)+\hat{a} \lim _{\delta \rightarrow 0} \frac{\beta(\delta)-\alpha(\delta)}{\delta}-\left(a \times \frac{\partial a}{\partial x_{k}}\right) \lim _{\delta \rightarrow 0} \gamma(\delta)
\end{aligned}
$$

So, we need to calculate the following three limits.

$$
(i)=\lim _{\delta \rightarrow 0} \beta(\delta),(i i)=\lim _{\delta \rightarrow 0} \frac{\beta(\delta)-\alpha(\delta)}{\delta},(i i i)=\lim _{\delta \rightarrow 0} \gamma(\delta)
$$

From (4.20) follows that we need to calculate asymptotics of $a_{1}(\delta), b_{1}(\delta), c_{1}(\delta), d(\delta)$, $\delta \rightarrow 0$. We have

$$
\begin{gathered}
\theta=|a|(x), \phi=|a|\left(x+\delta e_{k}\right)=|a|(x)+\delta \frac{\partial}{\partial x_{k}}|a|+\underline{o}(\delta), \\
\cos (\angle(u, v))=\frac{\left(-a(x), a\left(x+\delta e_{k}\right)\right)}{|a|(x)|a|\left(x+\delta e_{k}\right)}=-1+\bar{o}\left(\delta^{2}\right)
\end{gathered}
$$




$$
\begin{array}{r}
a_{1}=\sin |a|\left(\frac{1+\cos |a|\left(x+\delta e_{k}\right)}{2}\right)-\sin |a|\left(x+\delta e_{k}\right) \times \\
\left(\frac{1-\cos |a|(x)}{2}\right)\left(-1+\bar{o}\left(\delta^{2}\right)\right)= \\
\sin |a|\left(\frac{1+\cos \left(|a|+\delta \frac{\partial}{\partial x_{k}}|a|\right)}{2}\right)+\left(\frac{1-\cos |a|}{2}\right) \times \\
\sin \left(|a|+\delta \frac{\partial}{\partial x_{k}}|a|\right)+\bar{o}\left(\delta^{2}\right)= \\
\frac{\sin |a|}{2}\left(1+\cos |a|-\sin |a| \frac{\partial}{\partial x_{k}}|a| \delta\right)+ \\
\left(\frac{1-\cos |a|}{2}\right)\left(\sin |a|+\cos |a| \frac{\partial}{\partial x_{k}}|a| \delta\right)+\bar{o}\left(\delta^{2}\right)= \\
=\sin |a|(x)-\frac{1}{2}(1-\cos |a|) \frac{\partial}{\partial x_{k}}|a| \delta+\bar{o}\left(\delta^{2}\right)
\end{array}
$$

Similarly,

$$
\begin{array}{r}
b_{1}=\sin |a|\left(x+\delta e_{k}\right)\left(\frac{1+\cos |a|}{2}\right)-\sin |a| \times \\
\left(\frac{1-\cos |a|\left(x+\delta e_{k}\right)}{2}\right)\left(-1+\bar{o}\left(\delta^{2}\right)\right)= \\
\sin \left(|a|+\delta \frac{\partial}{\partial x_{k}}|a|\right)\left(\frac{1+\cos |a|}{2}\right)+ \\
\sin |a|\left(\frac{1-\cos \left(|a|+\delta \frac{\partial}{\partial x_{k}}|a|\right)}{2}\right)+\bar{o}\left(\delta^{2}\right)= \\
\left(\sin |a|+\cos |a| \frac{\partial}{\partial x_{k}}|a| \delta\right)\left(\frac{1+\cos |a|}{2}\right)+ \\
\frac{1}{2} \sin |a|\left(\cos |a|-\delta \sin |a| \frac{\partial}{\partial x_{k}}|a|\right)+\bar{o}\left(\delta^{2}\right)= \\
\sin |a|+\frac{1}{2}(1+\cos |a|) \frac{\partial}{\partial x_{k}}|a| \delta+\bar{o}\left(\delta^{2}\right) \\
c_{1}=1-\cos |a|+\bar{o}(\delta) \\
d=\bar{o}(\delta)
\end{array}
$$

From (4.27),(4.28),(4.29) and (4.30) we get

$$
\begin{gathered}
(i i i)=\lim _{\delta \rightarrow 0} \frac{\sin ^{-1}(d)}{d} \frac{c_{1}}{|a|(x)|a|\left(x+\delta e_{k}\right)}=\frac{1-\cos |a|}{|a|^{2}} \\
(i)=\lim _{\delta \rightarrow 0} \frac{\sin ^{-1}(d)}{d} \frac{b_{1}}{|a|\left(x+\delta e_{k}\right)}=\frac{\sin |a|}{|a|} \\
(i i)=\lim _{\delta \rightarrow 0} \frac{\sin ^{-1}(d)}{d} \frac{1}{\delta}\left(\frac{\sin |a|+\frac{1}{2}(1+\cos |a|) \frac{\partial}{\partial x_{k}}|a| \delta+\bar{o}\left(\delta^{2}\right)}{|a|\left(x+\delta e_{k}\right)}-\right. \\
\left.\frac{\sin |a|(x)-\frac{1}{2}(1-\cos |a|) \frac{\partial}{\partial x_{k}}|a| \delta+\bar{o}\left(\delta^{2}\right)}{|a|}\right)=\frac{|a|-\sin |a|}{|a|^{2}} \frac{\partial}{\partial x_{k}}|a|
\end{gathered}
$$


Thus, we get

$$
\begin{array}{r}
\exp (-\hat{a}) \frac{\partial}{\partial x_{k}} \exp (\hat{a})=\frac{\sin |a|}{|a|} \frac{\partial \hat{a}}{\partial x_{k}}+\frac{|a|-\sin |a|}{|a|^{2}} \frac{\partial}{\partial x_{k}}|a| \hat{a}+ \\
\frac{\cos |a|-1}{|a|^{2}} a \times \frac{\partial a}{\partial x_{k}}
\end{array}
$$

If we put $b=\frac{a}{|a|}$ and insert it in (4.32) we get (4.25).

Everywhere below we assume that $v \in L^{1}\left(0, T ; C_{b}^{2, \alpha}\left(\mathbb{R}^{n}, \mathbb{R}^{n}\right)\right)$ for some $\alpha \in(0,1)$.

COROLlary 4.21. Let $\left(X_{s}(t ; x)\right), 0 \leq s \leq t \leq T$ be the stochastic flow corresponding to

$$
\begin{aligned}
d X_{s}(t ; x) & =v\left(t, X_{s}(t ; x)\right) d t+\sqrt{2 \nu} \sigma_{1}\left(t, X_{s}(t ; x)\right) d W(t), \\
X_{s}(s ; x) & =x,
\end{aligned}
$$

where $\sigma_{1}(t, x)=\exp (\hat{a})(t, x), b=\frac{a}{|a|} \in S(2)$. Then for all $s, t \in[0, T]$ such that $s \leq t$,

$$
\begin{aligned}
& \int_{X_{T-s}(t ; \Gamma)} \sum_{k=1}^{n} F^{k}(T-t, x) d x_{k}=\int_{\Gamma} \sum_{k=1}^{n} F^{k}(s, x) d x_{k} \\
& +\int_{T-s X_{T-s}(\tau ; \Gamma)}^{t} \int_{k=1}^{n}\left(\frac{\partial F^{k}}{\partial t}+\sum_{j=1}^{n} v^{j}\left(\frac{\partial F^{k}}{\partial x_{j}}-\frac{\partial F^{j}}{\partial x_{k}}\right)+\nu \triangle F^{k}\right) d x_{k} d \tau
\end{aligned}
$$

(4.34) $+\nu \int_{T-s}^{t} \int_{X_{T-s}(\tau ; \Gamma)}\left(\operatorname{curl} F,(1-\cos |a|) b \times \frac{\partial b}{\partial x_{k}}+\sin |a| \frac{\partial b}{\partial x_{k}}+b \frac{\partial|a|}{\partial x_{k}}\right) d x_{k} d \tau$

$$
+\sqrt{2 \nu} \int_{T-s}^{t} \int_{X_{T-s}(\tau ; \Gamma)} \sum_{k=1}^{n}\left(\sum_{i, l=1}\left(\frac{\partial F^{k}}{\partial x_{i}}-\frac{\partial F^{i}}{\partial x_{k}}\right) \sigma_{1}^{i l}\right) d x_{k} d W_{\tau}^{l} .
$$

Proof OF COROLlary 4.21. Immediately follows from Proposition 4.18 and identity

$$
\sum_{i, j} \frac{\partial F^{i}}{\partial x_{j}}(\hat{a})^{i j}=(\operatorname{curl} F, a)
$$

REMARK 4.22. The vector $b$ can be interpreted as the axis of rotation of $\sigma$ and $\phi=|a|$ as the angle of rotation.

Now, we present a three dimensional analog of the two dimensional result from Proposition 4.9 .

Proposition 4.23. Assume that $F_{0} \in C_{0}^{\infty}\left(\mathbb{R}^{3}\right), v \in L^{1}\left(0, T ; C_{b}^{2, \alpha}\left(\mathbb{R}^{n}, \mathbb{R}^{n}\right)\right)$, $\alpha \in(0,1)$, $v$ satisfies condition (4.6), and $F \in L^{\infty}\left([0, T] ; C^{2+\delta}\left(\mathbb{R}^{3}, \mathbb{R}^{3}\right)\right)$ is a solution of equation (4.4)-(4.5) such that for some $\beta>0$ and any smooth closed loop $\Gamma$ condition (4.7) is satisfied. Let $\left(X_{s}(t ; x)\right), 0 \leq s \leq t \leq T$ be the stochastic flow corresponding to

$$
\begin{aligned}
d X_{s}(t ; x) & =v\left(t, X_{s}(t ; x)\right) d t+\sqrt{2 \nu} \sigma_{1}\left(t, X_{s}(t ; x)\right) d W(t), \\
X_{s}(s ; x) & =x,
\end{aligned}
$$


where $\sigma_{1}(t, x)=\exp (\hat{a}(t, x)), a=\operatorname{curl} F$. Fix $s \in[0, T]$ and define a functions $Q_{s}$ : $\mathbb{R}^{3} \rightarrow \mathbb{R}^{3}$ by $Q_{s}=\mathbb{E}\left(F_{0}\left(X_{T-s}(T ; x)\right) \nabla X_{T-s}(T ; x)\right)$.

Then, $Q_{s} \in L^{2}\left(\mathbb{R}^{3}, \mathbb{R}^{3}\right) \cap C^{\varepsilon}\left(\mathbb{R}^{3}, \mathbb{R}^{3}\right), 0<\varepsilon<\alpha$ and

$$
F(s, x)=\left[\mathrm{P}\left(Q_{s}\right)\right](x), x \in \mathbb{R}^{3}, s \in[0, T] .
$$

Proof of Proposition 4.23. In view of [16, Theorem 4.6.5, p.173] we infer that there exists solution $X_{s}(t ; x), 0 \leq s \leq t \leq T$ of problem (4.35) and $X_{s}(t ; x), 0 \leq s \leq t \leq$ $T$ is a flow of $C^{1}$-diffeomorphisms. Furthermore, it is $C^{1+\varepsilon}\left(\mathbb{R}^{n}, \mathbb{R}^{n}\right)$-valued process for any $0<\varepsilon<\delta$.

Moreover, it follows from Theorems 3.3.3, p.94 and 4.6.5, p. 173 therein that for all $s \in[0, T], Q_{s} \in C^{\varepsilon}\left(\mathbb{R}^{n}, \mathbb{R}^{n}\right), 0<\varepsilon<\delta$. Let us fix $s \in[0, T]$. We will show now that $Q_{s} \in L^{2}\left(\mathbb{R}^{n}, \mathbb{R}^{n}\right)$. Since by Corollary 4.6 .7 p. 175 of [16] that there exists a positive constant $C$ such that

$$
\sup _{x \in \mathbb{R}^{n}} \mathbb{E}\left|\nabla X_{T-s}(T ; x)\right|^{2} \leq C,
$$

by the Hölder inequality we infer that

$$
\begin{array}{r}
\int_{\mathbb{R}^{n}}\left|Q_{s}(x)\right|^{2} d x \leq \int_{\mathbb{R}^{n}} \mathbb{E}\left|F_{0}\left(X_{T-s}(T ; x)\right)\right|^{2} \mathbb{E}\left|\nabla X_{T-s}(T ; x)\right|^{2} d x \\
\leq C \int_{\mathbb{R}^{n}} \mathbb{E}\left|F_{0}\left(X_{T-s}(T ; x)\right)\right|^{2} d x .
\end{array}
$$

Now it follows from Girsanov Theorem that

$$
\int_{\mathbb{R}^{n}} \mathbb{E}\left|F_{0}\left(X_{T-s}(T ; x)\right)\right|^{2} d x=\int_{\mathbb{R}^{n}} \tilde{\mathbb{E}}\left(\left|F_{0}\left(x+\sqrt{2 \nu}\left(W_{T}-W_{T-s}\right)\right)\right|^{2} \mathcal{E}_{T-s}^{T}\right) d x
$$

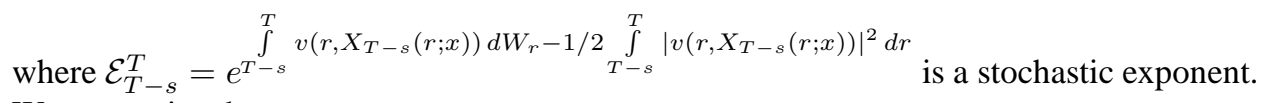
We can notice that

$$
\tilde{\mathbb{E}}\left|\mathcal{E}_{T-s}^{T}\right|^{2} \leq e^{2 \int_{0}^{T}|v(r)|_{L} \infty(r) d r}
$$

and, therefore, combining (4.37), (4.38) and (4.39) we get

$$
\int_{\mathbb{R}^{n}}\left|Q_{s}(x)\right|^{2} d x \leq e^{\int_{0}^{T}|v|_{L^{\infty}}(r) d r}\left(\int_{\mathbb{R}^{n}}\left|F_{0}\right|^{4} d x\right)^{\frac{1}{2}} .
$$

Now let us show that $\int_{X_{T-s}(t ; \Gamma)} \sum_{k=1}^{3} F^{k}(T-t) d x_{k}, t \in[T-s, T]$ is a local martingale. It is enough to prove that the "correction" term (due to nontrivial $\sigma_{1}$ ) in the formula (4.34) disappears.

Since $b=\frac{\operatorname{curl} F}{|\operatorname{curl} F|},|b|=1,|a|=|\operatorname{curl} F|$ we have

$$
\left(\operatorname{curl} F, \frac{\partial b}{\partial x_{k}}\right)=|\operatorname{curl} F|\left(b, \frac{\partial b}{\partial x_{k}}\right)=0 .
$$

Similarly,

$$
\left(\operatorname{curl} F, b \times \frac{\partial b}{\partial x_{k}}\right)=|\operatorname{curl} F|\left(b, b \times \frac{\partial b}{\partial x_{k}}\right)=0,
$$


and

$$
(\operatorname{curl} F, b) \frac{\partial|\operatorname{curl} F|}{\partial x_{k}}=\frac{1}{2} \frac{\partial|\operatorname{curl} F|^{2}}{\partial x_{k}} .
$$

QUESTION 4.24. It would be interesting to generalize Theorem 4.12 to the three dimensional case. In view of Corollary 4.21 in order to find such generalization it is enough to prove that for any solution $F$ of equation (4.4) with $v$ being the corresponding $\left.C^{\infty}\right)$ vector field, there exists a triple $(b, \phi, \psi) \in$ $\left(L^{\infty}\left([0, T], C^{\infty}\left(\mathbb{R}^{3}, S^{2}\right)\right), L^{\infty}\left([0, T], C^{\infty}\left(\mathbb{R}^{3}, S^{1}\right)\right), L^{\infty}\left([0, T], C^{\infty}\left(\mathbb{R}^{3}, \mathbb{R}\right)\right)\right)$ such that

$$
\begin{aligned}
(\cos \phi-1)\left(\operatorname{curl} F, b \times \frac{\partial b}{\partial x_{k}}\right) & +\sin \phi\left(\operatorname{curl} F, \frac{\partial b}{\partial x_{k}}\right) \\
+(\operatorname{curl} F, b) \frac{\partial \phi}{\partial x_{k}} & +\frac{\partial \psi}{\partial x_{k}}=\frac{(v \times \operatorname{curl} F)^{k}}{\nu}, k=1,2,3 .
\end{aligned}
$$

We can notice that system (4.41) is time independent in the sense that there are no time derivatives of the unknown functions. Therefore it is enough to consider the system for every fixed time $t \in[0, T]$. If the solenoidal vector field $v$ is two dimensional, i.e. $\operatorname{div} v=$ $0, v_{3}=0$ and the components $v_{1}, v_{2}$ do not depend upon variable $x_{3}$, then $b=(0,0,1)$, $\phi=\phi_{1} / \nu$, where $\phi_{1}$ is a stream function for $v, \psi=0$, is a solution of the system (4.41), see Theorem 4.12. However, in the three dimensional case the problem is completely open. One of the possibilities to narrow the problem is to consider the case when $F=u$ is a solution to the Navier-Stokes equations.

QUESTION 4.25. Another question connected with system (4.41) is as follows. How do variables $b, \phi, \psi$ depend upon $\nu$ ? Can one take the $\nu$ to 0 limit in the representation (4.41)? But let us note that in the two dimensional case under the additional condition of incompressibility $\operatorname{div} v=0$, the representation (4.41) holds also in the limit $\nu \rightarrow 0$. Indeed, in two dimensional case the stream function corresponding to the vector field $v$ exists because $\operatorname{div} v=0$ and is independent of $F$ and $\nu$.

REMARK 4.26. The Question 4.24 can be reformulated in the following way.

Problem A. Find a $C^{1}$-class function $\sigma: \mathbb{R}^{3} \rightarrow S O(3)$ such that for any smooth closed loop $\Gamma$

$$
\sum_{k} \int_{\Gamma} \sum_{j} v^{j}\left(\frac{\partial F^{k}}{\partial x_{j}}-\frac{\partial F^{j}}{\partial x_{k}}\right) d x_{k}=\nu \sum_{k=1}^{n} \int_{\Gamma} \sum_{j, l, m} \frac{\partial F^{j}}{\partial x_{l}} \sigma^{l m} \frac{\partial \sigma^{j m}}{\partial x_{k}} d x_{k} .
$$

Let $\wedge$ be the wedge product, see e.g. [30, p.79], and denote

$$
\begin{aligned}
\alpha & =\frac{1}{\nu} \sum_{j, k} v^{j}\left(\frac{\partial F^{k}}{\partial x_{j}}-\frac{\partial F^{j}}{\partial x_{k}}\right) d x_{k}, \\
w & =\operatorname{curl} F .
\end{aligned}
$$

Suppose $\sigma: \mathbb{R}^{3} \rightarrow S O(3)$ is a $C^{1}$-class function. Let us define a matrix valued function $A$,

$$
A=d \sigma \sigma^{-1}
$$

Then the matrix $A$ is antisymmetric and has the following form

$$
A=\left(\begin{array}{ccc}
0 & -a_{3} & a_{2} \\
a_{3} & 0 & -a_{1} \\
-a_{2} & a_{1} & 0
\end{array}\right)
$$


where $a_{i}(x), i=1,2,3, x \in \mathbb{R}^{3}$ are 1 -forms. Moreover, $A$ satisfies system

$$
d A+A \wedge A=0,
$$

or, in terms of 1 -forms $a_{i}, i=1,2,3$, equivalently

$$
\begin{aligned}
d a_{1} & =a_{3} \wedge a_{2} \\
d a_{2} & =a_{1} \wedge a_{3} \\
d a_{3} & =a_{2} \wedge a_{1} .
\end{aligned}
$$

Furthermore, if arbitrary antisymmetric matrix $A$ of one-forms satisfies (4.45) then there exists $\sigma: \mathbb{R}^{3} \rightarrow S O(3)$ such that (4.43) is satisfied. Notice that the right part of formula (4.42) can be rewritten as follows

$$
\nu \int_{\Gamma} \sum_{i=1}^{3} w_{i} a_{i}
$$

Indeed,

$$
\sum_{k, m} \sigma^{\cdot m} \frac{\partial \sigma^{\cdot m}}{\partial x_{k}} d x_{k}=d \sigma \sigma^{\perp}=d \sigma \sigma^{-1}=A .
$$

Now we can rewrite formula (4.42) as follows

$$
\int_{\Gamma} \alpha=-\int_{\Gamma} \sum_{i=1}^{3} w_{i} a_{i}
$$

Hence, we can reformulate the equation (4.41) as follows

$$
\sum_{i=1}^{3} w_{i} a_{i}=-\alpha+d \psi
$$

Thus, Problem A can be solved in two stages. First, we need to solve system

$$
\left\{\begin{aligned}
d a_{1} & =a_{3} \wedge a_{2} \\
d a_{2} & =a_{1} \wedge a_{3} \\
d a_{3} & =a_{2} \wedge a_{1} \\
\sum_{i=1}^{3} w_{i} a_{i} & =-\alpha+d \psi
\end{aligned}\right.
$$

Then we need to find $\sigma: \mathbb{R}^{3} \rightarrow S O(3)$ from equation (4.43). Existence of such $\sigma$ follows from first three equations of system (4.49).

Applying the exterior derivative operator $d$ to the last equation of the system (4.49) we can get rid of function $\psi$ and thus we get equivalent system

$$
\left\{\begin{aligned}
d a_{1} & =a_{3} \wedge a_{2} \\
d a_{2} & =a_{1} \wedge a_{3} \\
d a_{3} & =a_{2} \wedge a_{1} \\
-d \alpha & =\sum_{i=1}^{3} d w_{i} \wedge a_{i}+w_{1} a_{3} \wedge a_{2}+w_{2} a_{1} \wedge a_{3}+w_{3} a_{2} \wedge a_{1} .
\end{aligned}\right.
$$

This system can be reformulated in terms of matrix-valued 1-form $A$ as follows:

$$
\left\{\begin{aligned}
d A+A \wedge A & =0 \\
\operatorname{tr}(W A \wedge A+d W \wedge A) & =2 d \alpha,
\end{aligned}\right.
$$


where

$$
W=\left(\begin{array}{ccc}
0 & -w_{3} & w_{2} \\
w_{3} & 0 & -w_{1} \\
-w_{2} & w_{1} & 0
\end{array}\right)
$$

Thus we have quadratic equation on the space of flat connections.

Another application of Proposition 4.1 is a Feynman-Kac type formula for solutions of the following equation

(4.52) $\frac{\partial F}{\partial t}=-\nu A_{0} F+(v(T-\cdot) \cdot \nabla) F-(F \cdot \nabla) v(T-\cdot), t>0, x \in \mathbb{R}^{n}$,

(4.53) $F(0)=F_{0}$,

where $A_{0}$ is a Stokes operator, $F_{0} \in H$ and $v$ satisfies condition (3.5). For the simplicity sake we formulate the result for $n=3$.

Proposition 4.27. Let $v \in L^{1}\left(0, T ; C_{b}^{2, \alpha}\left(\mathbb{R}^{n}, \mathbb{R}^{n}\right)\right)$ for some $\alpha \in(0,1)$, $v$ satisfies condition (4.6), $\left(X_{s}(t ; x)\right), 0 \leq s \leq t<\infty$ is the flow corresponding to problem (4.3), $F_{0} \in C_{0}^{\infty}\left(\mathbb{R}^{n}\right)$ and $F$ is a solution of equation (4.52) such that there exists $\beta>0$ : (4.54)

$$
\mathbb{E}\left|\int_{X_{T-s}(t ; S)} F^{1}(T-t, x) d x_{2} d x_{3}+F^{2}(T-t, x) d x_{3} d x_{1}+F^{3}(T-t, x) d x_{1} d x_{3}\right|^{1+\beta}<\infty
$$

for any smooth surface $S \subset \mathbb{R}^{3}$ with smooth boundary $\Gamma$ and all $0 \leq T-s \leq t \leq T$. Then it satisfies

$$
\begin{aligned}
& F^{1}(s, x)= \\
& \mathbb{E}\left[F_{0}^{1}\left(X_{T-s}(T ; x)\right)\left(\frac{\partial X_{T-s}^{2}(T ; x)}{\partial x_{2}} \frac{\partial X_{T-s}^{3}(T ; x)}{\partial x_{3}}-\frac{\partial X_{T-s}^{2}(T ; x)}{\partial x_{3}} \frac{\partial X_{T-s}^{3}(T ; x)}{\partial x_{2}}\right)\right. \\
&(4.55)+ F_{0}^{2}\left(X_{T-s}(T ; x)\right)\left(\frac{\partial X_{T-s}^{3}(T ; x)}{\partial x_{2}} \frac{\partial X_{T-s}^{1}(T ; x)}{\partial x_{3}}-\frac{\partial X_{T-s}^{3}(T ; x)}{\partial x_{3}} \frac{\partial X_{T-s}^{1}(T ; x)}{\partial x_{2}}\right) \\
&+F_{0}^{3}\left(X_{T-s}(T ; x)\right)\left(\frac{\partial X_{T-s}^{1}(T ; x)}{\partial x_{2}} \frac{\partial X_{T-s}^{2}(T ; x)}{\partial x_{3}}-\frac{\partial X_{T-s}^{1}(T ; x)}{\partial x_{3}} \frac{\partial X_{T-s}^{2}(T ; x)}{\partial x_{2}}\right) \\
& F^{2}(s, x)= \\
& \mathbb{E}\left[F_{0}^{1}\left(X_{T-s}(T ; x)\right)\left(\frac{\partial X_{T-s}^{2}(T ; x)}{\partial x_{3}} \frac{\partial X_{T-s}^{3}(T ; x)}{\partial x_{1}}-\frac{\partial X_{T-s}^{2}(T ; x)}{\partial x_{1}} \frac{\partial X_{T-s}^{3}(T ; x)}{\partial x_{3}}\right)\right. \\
&+F_{0}^{3}\left(X_{T-s}(T ; x)\right)\left(\frac{\partial X_{T-s}^{1}(T ; x)}{\partial x_{3}} \frac{\partial X_{T-s}^{2}(T ; x)}{\partial x_{1}}-\frac{\partial X_{T-s}^{1}(T ; x)}{\partial x_{1}} \frac{\partial X_{T-s}^{2}(T ; x)}{\partial x_{3}}\right) \\
& F^{3}(s, x)= \\
& \mathbb{E}\left[F_{0}^{1}\left(X_{T-s}(T ; x)\right)\left(\frac{\partial X_{T-s}^{2}(T ; x)}{\partial x_{1}} \frac{\partial X_{T-s}^{3}(T ; x)}{\partial x_{2}}-\frac{\partial X_{T-s}^{2}(T ; x)}{\partial x_{2}} \frac{\partial X_{T-s}^{3}(T ; x)}{\partial x_{1}}\right)\right. \\
&+ F_{0}^{3}\left(X_{T-s}(T ; x)\right)\left(\frac{\partial X_{T-s}^{1}(T ; x)}{\partial x_{1}} \frac{\partial X_{T-s}^{2}(T ; x)}{\partial x_{2}}-\frac{\partial X_{T-s}^{1}(T ; x)}{\partial x_{2}} \frac{\partial X_{T-s}^{2}(T ; x)}{\partial x_{1}}\right) \\
& \text { (4.57) }+F_{0}^{2}\left(X_{T-s}(T ; x)\right)\left(\frac{\partial X_{T-s}^{3}(T ; x)}{\partial x_{1}} \frac{\partial X_{T-s}^{1}(T ; x)}{\partial x_{2}}-\frac{\partial X_{T-s}^{3}(T ; x)}{\partial x_{T-s}^{1}(T ; x)}\right)
\end{aligned}
$$


Proof of Proposition 4.27. The result follows from Proposition 4.5. Indeed, let $G \in L^{\infty}\left(0, T ; L^{2}\left(\mathbb{R}^{n}, \mathbb{R}^{n}\right) \cap C^{1+\varepsilon}\left(\mathbb{R}^{n}, \mathbb{R}^{n}\right)\right), 0<\varepsilon<\alpha$ be a solution of equation (4.4)-(4.5). Its existence follows from Proposition 4.5. Then $F=\operatorname{curl} G$ is a solution of equation (4.52). For solution $G$ of (4.4) we have got representation by formula (4.8) of Feynman-Kac type. Integrating it w.r.t. closed contour $\Gamma$ we get

$$
\int_{\Gamma} \sum_{k} G^{k}(s, x) d x_{k}=\mathbb{E}\left(\int_{X_{T-s}(T ; \Gamma)} \sum_{k} G_{0}^{k}(x) d x_{k}\right) .
$$

Now, result immediately follows from Stokes Theorem.

REMARK 4.28. On an informal level, the Feynman-Kac type formula (4.55)-(4.57) in the case of $\nu=0$ can be seen as a solution of the following informal infinite dimensional first order PDE obtained by the characteristics method. Indeed, let us denote by $Y$ the set of all smooth surfaces $S \subset \mathbb{R}^{n}$ with smooth boundary $\Gamma$. Let $T Y$ be the set of all smooth vector fields on $Y$. If $F$ is a solution of equation (4.52) with parameters $\nu=0$ and $v \in C_{0}^{\infty}\left([0, T] \times \mathbb{R}^{n}\right)$, then $\tilde{F}$ defined by

$$
\tilde{F}:[0, \infty) \times Y \ni(t, S) \mapsto \int_{S}(F(t, \cdot), \vec{n}) d \sigma \in \mathbb{R},
$$

is a solution to the following equation

$$
\frac{\partial \tilde{F}}{\partial t}=D_{\tilde{v}} \tilde{F}
$$

where $D_{\tilde{v}}$ is directional derivative along the vector field $\tilde{v} \in T Y$ defined by

$$
Y \ni S \mapsto \bigcup_{x \in S} v(x) \in T Y
$$

Then, on a purely speculative level, the solution to equation (4.59) obtained via the characteristics method is exactly our Feynman-Kac type formula.

REMARK 4.29. In a forthcoming publication the authors will consider the case of equations with less regular velocity vector fields than those considered in the current paper. Transport equations with irregular velocity field have been a subject of a great variety of works, see e.g. recent works by Lions and Di Perna [7], Maniglia [21], Bouchot, James and Mancini [2], and references therein. Our plan is to combine the results of Maniglia [21] with our work i.e. to find probabilistic representation of solution of vector advection equation with irregular velocity and then study the limit as the viscosity $\nu$ converges to 0 .

\section{Proofs of results from section 3}

Proof of Proposition 3.2.(i) The proof will be divided into three parts a), b), c). a) Let us consider a special case when $v \in L^{\infty}\left(0, T ; \mathbb{L}^{3+\delta_{0}}(D)\right)$. We will use Theorem 2.3 with Gelfand triple $V \subset H \cong H^{\prime} \subset V^{\prime}$. Denote $A(t)=\nu A+B(v(t), \cdot)$. We need to check whether the conditions (2.5) and (2.6) are satisfied. We have,

$$
\langle A(t) f, f\rangle_{V^{\prime}, V}=\nu \tilde{a}(f, f)+\langle B(v(t), f), f\rangle_{V^{\prime}, V}, f \in V
$$

The second term on the RHS of the equality (5.1) from (2.12) can be estimated as follows

$$
\begin{array}{r}
\left|\langle B(v(t), f), f\rangle_{V^{\prime}, V}\right| \leq \frac{1}{2}\|f\|_{V}^{2}+\frac{1}{2}\left(\varepsilon^{1+\delta_{0} / 3}\|f\|_{V}^{2}\right. \\
\left.+\frac{C_{\delta_{0}}}{\varepsilon^{1+3 / \delta_{0}}}|v(t)|_{\mathbb{L}^{3+\delta_{0}(D)}}^{2+\frac{6}{\delta_{0}}}|f|_{H}^{2}\right), \varepsilon>0 .
\end{array}
$$


Thus from the inequality (5.2) and the continuity of form $\tilde{a}$ we infer that,

$$
\|A(t)\|_{\mathcal{L}\left(V, V^{\prime}\right)} \leq C \nu+C_{2}|v(t)|_{\mathbb{L}^{3+\delta_{0}}(D)}
$$

The coercivity assumption (2.6) also follows from the inequality (2.12). Indeed, for $f \in V$, $t \in[0, T]$ we have

$$
\begin{gathered}
\left|\langle A(t) f, f\rangle_{V^{\prime}, V}\right|=\left|\nu \tilde{a}(f, f)+\langle B(v(t), f), f\rangle_{V^{\prime}, V}\right| \geq \\
\frac{\nu}{2}\|f\|_{V}^{2}-\frac{C}{\nu}\left(\varepsilon^{1+\delta_{0} / 3}\|f\|_{V}^{2}+\frac{C_{\delta_{0}}}{\varepsilon^{1+3 / \delta}}|v(t)|_{\mathbb{L}^{3+\delta_{0}(D)}}^{2+\frac{6}{\delta_{0}}}|f|_{H}^{2}\right) .
\end{gathered}
$$

By choosing $\varepsilon>0$ such that $\frac{\nu}{2}-\frac{C}{\nu} \varepsilon^{1+\delta_{0} / 3}>0$ we conclude the proof of the coercivity condition (2.6). Thus, by the Theorem 2.3, first statement of the Proposition follows.

b) To prove Proposition in the general case we will show an energy inequality for solutions of equation (3.1-3.2) when $v \in L^{\infty}\left(0, T ; \mathbb{L}^{3+\delta_{0}}(D)\right)$. From step (a) we know that a solution $F \in L^{2}(0, T ; V)$ such that $F^{\prime} \in L^{2}\left(0, T ; V^{\prime}\right)$ exists and unique. Then, from Lemma 2.1 and equality (2.18) we infer that

$$
\begin{aligned}
\frac{1}{2} \frac{d}{d t}|F|_{H}^{2} & =-\nu\|F\|_{V}^{2}+\langle f, F\rangle_{V^{\prime}, V}-\langle B(v, F), F\rangle_{V^{\prime}, V} \\
& =-\nu\|F\|_{V}^{2}+\langle f, F\rangle_{V^{\prime}, V}+(\operatorname{curl} F, v \times F)_{H}
\end{aligned}
$$

Therefore, by applying the Young inequality, we infer that

$$
\begin{aligned}
|F(t)|_{H}^{2} & +2 \nu \int_{0}^{t}|F(s)|_{V}^{2} d s-\int_{0}^{t}(\operatorname{curl} F(s), v(s) \times F(s))_{H} d s \\
& =|F(0)|_{H}^{2}+\int_{0}^{t}\langle f(s), F(s)\rangle_{V^{\prime}, V} d s \\
& \leq|F(0)|_{H}^{2}+\frac{\nu}{2} \int_{0}^{t}|F(s)|_{V}^{2} d s+\frac{C}{\nu} \int_{0}^{t}|f(s)|_{V^{\prime}}^{2} d s .
\end{aligned}
$$


The term $\int_{0}^{t}(\operatorname{curl} F(s), v(s) \times F(s))_{H} d s$ can be estimated as follows:

$$
\begin{aligned}
& \left|\int_{0}^{t}(\operatorname{curl} F(s), v(s) \times F(s))_{H} d s\right| \\
\leq & \frac{\nu}{4} \int_{0}^{t}|\operatorname{curl} F|_{H}^{2} d s+\frac{C}{\nu} \int_{0}^{t}|v(s) \times F(s)|_{H}^{2} d s \\
\leq & \frac{\nu}{4} \int_{0}^{t}|\operatorname{curl} F|_{H}^{2} d s+\frac{C}{\nu} \int_{0}^{t}\left(\varepsilon^{1+\delta_{0} / 3}|F(s)|_{V}^{2}\right. \\
& \left.+\frac{C_{\delta_{0}}}{\varepsilon^{1+\delta_{0} / 3}}|v(s)|_{\mathbb{L}^{3+\delta_{0}}}^{2+6 / \delta_{0}}|F(s)|_{H}^{2}\right) d s \\
\leq \quad & \left.\frac{\nu}{4}+\frac{C}{\nu} \varepsilon^{1+\delta_{0} / 3}\right) \int_{0}^{t}|F(s)|_{V}^{2} d s \\
& +\frac{C_{\delta_{0}}}{\nu \varepsilon^{1+\delta_{0} / 3}} \int_{0}^{t}|v(s)|_{\mathbb{L}^{3+\delta_{0}}}^{2+6 / \delta_{0}}|F(s)|_{H}^{2} d s .
\end{aligned}
$$

Let us choose $\varepsilon>0$ such that $\frac{\nu}{4}+\frac{C}{\nu} \varepsilon^{1+\delta_{0} / 3}=\frac{\nu}{2}$. Then

$$
\begin{array}{r}
|F(t)|_{H}^{2}+\nu \int_{0}^{t}\|F(s)\|_{V}^{2} d s \leq|F(0)|_{H}^{2}+\frac{C}{\nu} \int_{0}^{t}|f(s)|_{V^{\prime}}^{2} d s \\
\quad+\frac{C_{\delta_{0}}}{\nu \varepsilon^{1+\delta_{0} / 3}} \int_{0}^{t}|v(s)|_{\mathbb{L}^{3+\delta_{0}}}^{2+6 / \delta_{0}}|F(s)|_{H}^{2} d s, t \geq 0 .
\end{array}
$$

Hence, in view of the Gronwall Lemma, we get

$$
|F(t)|_{H}^{2} \leq\left(|F(0)|_{H}^{2}+\frac{C}{\nu} \int_{0}^{t}|f(s)|_{V^{\prime}}^{2} d s\right) e^{C\left(\delta_{0}, \nu\right) \int_{0}^{t}|v(s)|_{\mathbb{L}^{3+\delta_{0}}}^{2+6 / \delta_{0}} d s}, t \geq 0 .
$$

Thus

$$
\begin{aligned}
|F(t)|_{H}^{2}+ & \nu \int_{0}^{t}\|F(s)\|_{V}^{2} d s \leq K_{1}\left(|F(0)|_{H}^{2}+\frac{C}{\nu} \int_{0}^{t}|f(s)|_{V^{\prime}}^{2} d s\right) \\
& \left(1+\int_{0}^{t}|v(s)|_{\mathbb{L}^{3+\delta_{0}}}^{2+6 / \delta_{0}} d s\right) e^{C\left(\delta_{0}, \nu\right) \int_{0}^{t}|v(s)|_{\mathbb{L}^{3+\delta_{0}}}^{2+6 / \delta_{0}} d s}, t \geq 0 .
\end{aligned}
$$

(c) The general case. Let $v_{n} \in L^{\infty}\left(0, T ; \mathbb{L}^{3+\delta_{0}}(D)\right)$ be a sequence of functions such that $v_{n} \rightarrow v$ in $L^{2+\frac{6}{\delta_{0}}}\left(0, T ; \mathbb{L}^{3+\delta_{0}}(D)\right)$. Let $F_{n}$ be a corresponding sequence of solutions of equation (3.1-3.2) with $v$ being replaced by $v_{n}$. Then from inequality (5.5) it follows that the sequence $\left\{F_{n}\right\}_{n=1}^{\infty}$ lies in a bounded set of $L^{\infty}(0, T ; H) \cap L^{2}(0, T ; V)$. Therefore, 
by the Banach-Alaoglu Theorem there exists subsequence $\left\{F_{n^{\prime}}\right\}$ and $F^{*} \in L^{\infty}(0, T ; H)$ such that for any $q \in L^{1}(0, T ; H)$

$$
\int_{0}^{T}\left(F_{n^{\prime}}-F^{*}, q(s)\right)_{H} d s \rightarrow 0
$$

Similarly, from the Banach-Alaoglu Theorem it follows that we can find a subsequence $\left\{F_{n^{\prime \prime}}\right\}$ of $\left\{F_{n^{\prime}}\right\}$ convergent to $F^{* *} \in L^{2}(0, T ; V)$ weakly i.e. for any $q \in L^{2}\left(0, T ; V^{\prime}\right)$

$$
\int_{0}^{T}\left\langle F_{n^{\prime \prime}}-F^{* *}, q(s)\right\rangle_{V^{\prime}, V} d s \rightarrow 0,
$$

In particular, (5.6) and (5.7) are satisfied for $q \in L^{2}(0, T ; H)$. Therefore $F^{*}=F^{* *} \in$ $L^{\infty}(0, T ; H) \cap L^{2}(0, T ; V)$. Put $F=F^{*}$. Let us now show that $F$ satisfies equation (3.1-3.2) in the weak sense. Let $\psi \in C^{\infty}([0, T], \mathbb{R}), \psi(1)=0, h \in V$. Then by part $(a)$ of the proof we have

$$
\begin{aligned}
-\int_{0}^{T}\left(F_{n}(s), h\right)_{H} \psi^{\prime}(s) d s & +\int_{0}^{T}\left\langle B\left(v_{n}, F_{n}\right), h\right\rangle_{V^{\prime}, V} \psi(s) d s+\nu \int_{0}^{T} \tilde{a}\left(F_{n}(s), h\right) \psi(s) d s \\
& =\left(F_{0}, h\right)_{H} \psi(0)+\int_{0}^{T}\langle f(s), h\rangle_{V^{\prime}, V} \psi(s) d s .
\end{aligned}
$$

Convergence of the first term, respectively third term, follows immediately from (5.6), respectively (5.7). For the second term we have

$$
\begin{array}{r}
\left|\int_{0}^{T}\left\langle B\left(v_{n}, F_{n}\right)-B(v, F), h\right\rangle_{V^{\prime}, V} \psi(s) d s\right| \leq\left|\int_{0}^{T}\left\langle B\left(v_{n}-v, F_{n}\right), h\right\rangle_{V^{\prime}, V} \psi(s) d s\right| \\
+\left|\int_{0}^{T}\left\langle B\left(v, F_{n}-F\right), h\right\rangle_{V^{\prime}, V} \psi(s) d s\right|=I_{n}+I I_{n} .
\end{array}
$$

Let $\varepsilon>0$ be fixed. For any $\varepsilon_{2}, \varepsilon_{3}>0$ we have, by inequality (2.12), the following inequalities

$$
\begin{aligned}
I_{n} & \leq \varepsilon_{3} \int_{0}^{T}\left|\operatorname{curl} F_{n}\right|_{H}^{2} d s+\frac{C}{\varepsilon_{3}} \int_{0}^{T}\left(\varepsilon_{2}|h|_{V}^{2}+\frac{C}{\varepsilon_{2}}\left|v_{n}-v\right|_{\mathbb{L}^{3+\delta_{0}}(D)}^{2+\frac{6}{\delta_{0}}}|h|_{H}^{2}\right)|\psi|^{2} d s \\
& =\varepsilon_{3}\left\|F_{n}\right\|_{L^{2}(0, T ; V)}^{2}+\frac{C \varepsilon_{2}}{\varepsilon_{3}}|h|_{V}^{2} \int_{0}^{T}|\psi|^{2} d s+\frac{C|h|_{H}^{2}}{\varepsilon_{3} \varepsilon_{2}} \int_{0}^{T}\left|v_{n}-v\right|_{\mathbb{L}^{3+\delta_{0}(D)}}^{2+\frac{6}{\delta_{0}}}|\psi|^{2} d s .
\end{aligned}
$$

Taking into account boundedness of the sequence $\left\{F_{n}\right\}_{n=1}^{\infty}$ in $L^{2}(0, T ; V)$ and the convergence of $\left\{v_{n}\right\}_{n=1}^{\infty}$ to $v$ in $L^{2+\frac{6}{\delta_{0}}}\left(0, T ; \mathbb{L}^{3+\delta_{0}}(D)\right)$, we can choose $\varepsilon_{2}, \varepsilon_{3}$ and $N=N(\varepsilon)$ in such way that $I_{n} \leq \frac{\varepsilon}{2}$, for $n \geq N$.

For $I I_{n}$ we have $I I_{n}=\left|\int_{0}^{T}\left\langle F_{n}-F, \operatorname{curl}(v \times h)\right\rangle_{V^{\prime}, V} \psi(s) d s\right|$. From inequality (2.14) it follows that $v \times h \in L^{2}(0, T ; H)$. Therefore, $\operatorname{curl}(v \times h) \in L^{2}\left(0, T ; V^{\prime}\right)$ and the convergence of $I I_{n}$ to 0 follows from inequality (5.7). The uniqueness of $F$ follows from 
the energy inequality (5.5). It remains to show that $F \in C\left([0, T], H_{w}\right)$. Let us show that $F \in C\left([0, T], V^{\prime}\right)$. Then, since $F \in L^{\infty}(0, T ; H)$, it immediately follows from [33, Lemma 1.4, p.178] that $F \in C\left([0, T], H_{w}\right)$. To prove that $F \in C\left([0, T], V^{\prime}\right)$ it is enough to show that $F^{\prime} \in L^{1}\left(0, T ; V^{\prime}\right)$. Indeed, we have that $F \in L^{\infty}(0, T ; H) \subset L^{1}\left(0, T ; V^{\prime}\right)$ and by [33, Lemma 1.1, p.169] the result follows. We have

$$
\begin{aligned}
& \left|F^{\prime}\right|_{L^{1+\frac{3}{2 \delta_{0}+3}}}^{1+\frac{3}{2 \delta_{0}+3}}\left(0, T ; V^{\prime}\right)
\end{aligned}
$$

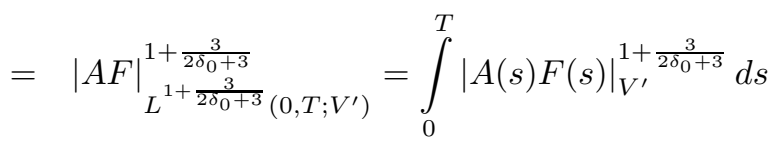

$$
\begin{aligned}
& \leq \int_{0}^{T}|A(s)|_{\mathcal{L}\left(V, V^{\prime}\right)}^{1+\frac{3}{2 \delta_{0}+3}}|F(s)|_{V}^{1+\frac{3}{2 \delta_{0}+3}} d s \\
& \leq \quad\left(\int_{0}^{T}|F(s)|_{V}^{2} d s\right)^{\frac{\delta_{0}+3}{2 \delta_{0}+3}}\left(\int_{0}^{T}|A(s)|_{\mathcal{L}\left(V, V^{\prime}\right)}^{2+\frac{6}{\delta_{0}}} d s\right)^{\frac{\delta_{0}}{2 \delta_{0}+3}} \\
& \leq\left(\int_{0}^{T}|F(s)|_{V}^{2} d s\right)^{\frac{\delta_{0}+3}{2 \delta_{0}+3}}\left(\int_{0}^{T}\left(C_{1} \nu+C_{2}|v(s)|_{\mathbb{L}^{3+\delta_{0}}(D)}\right)^{2+\frac{6}{\delta_{0}}} d s\right)^{\frac{\delta_{0}}{2 \delta_{0}+3}}
\end{aligned}
$$

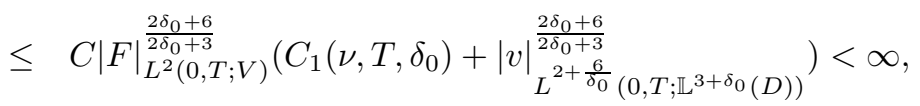

where the second inequality follows from the Hölder inequality and the third one follows from the inequality (5.3). Thus, first statement of the Proposition 3.2 is proved.

(ii) To prove [ii] we follow an idea from [3] and [4].

Lemma 5.1. Let $g:[0, T] \rightarrow \mathbb{R}$ be measurable function such that $\int_{0}^{T}|g(s)| d s<$ $\infty$. Then for any $\delta>0$ there exists a partition $\left\{T_{i}\right\}_{i=1}^{n}$ of interval $[0, T]$ such that $\int_{T_{i}}^{T_{i+1}}|g(s)| d s<\delta, i=1, \ldots, n$.

PROOF. Follows easily from [28, Theorem 8.17].

Existence of a local solution. Let $X_{T}=\left\{F \in L^{2}(0, T ; D(A)): F^{\prime} \in L^{2}(0, T ; H)\right\}$ be a Banach space endowed with a norm

$$
|F|_{X_{T}}^{2}=\nu^{2}|F|_{L^{2}(0, T ; D(A))}^{2}+\left|F^{\prime}\right|_{L^{2}(0, T ; H)}^{2} .
$$

We will prove the following result.

Lemma 5.2. If $v$ satisfies assumption (3.5), $z \in X_{T}$ then $B(v(\cdot), z) \in L^{2}(0, T ; H)$.

In view of Proposition 2.4 and the above Lemma, a map $\Phi_{T}: X_{T} \rightarrow X_{T}$ defined by $\Phi_{T}(z)=G$ iff $G$ is the unique solution solution of the problem

$$
G^{\prime}+\nu A G=f-B(v(t), z), G(0)=F_{0},
$$

is well defined. 
Proof OF LemMA 5.2. From inequality (2.20) we have

$$
\begin{aligned}
& \|B(v(\cdot), z)\|_{L^{2}(0, T ; H)}^{2} \leq C_{1}\left(\varepsilon, \delta_{0}\right)\|z\|_{L^{2}\left(0, T ; \mathbb{H}^{2}(D)\right)}^{2} \\
& +C_{2}\left(\varepsilon, \delta_{0}\right)|z|_{C([0, T] ; V)}^{2}|v|_{L^{2+\frac{6}{\delta_{0}}}}{ }_{\left(0, T ; \mathbb{L}^{3+\delta_{0}}(D)\right)}
\end{aligned}
$$

Thus the result follows from Lemma 2.1.

We will show that there exists $T_{1} \leq T$ such that $\Phi_{T_{1}}$ is a strict contraction. By Proposition 2.4 and inequality (2.20) we have, for all $t \in[0, T]$,

$$
\begin{aligned}
& \left\|\Phi_{t}\left(z_{1}\right)-\Phi_{t}\left(z_{2}\right)\right\|_{X_{t}}^{2} \\
& \leq C_{1}\left\|B\left(v, z_{1}-z_{2}\right)\right\|_{L^{2}(0, t ; H)}^{2} \leq C_{1} \varepsilon^{1+\delta_{0} / 3}\left|z_{1}-z_{2}\right|_{L^{2}(0, t ; D(A))}^{2} \\
& +\quad C_{1} \frac{C_{\delta}}{\varepsilon^{1+3 / \delta}}\left|z_{1}-z_{2}\right|_{C(0, t ; V)}^{2}|v|_{L^{2+6 / \delta_{0}}\left(0, T ; \mathbb{L}^{3+\delta_{0}}(D)\right)} \\
& \leq C_{1} \varepsilon^{1+\delta_{0} / 3}\left|z_{1}-z_{2}\right|_{X_{t}}^{2} \\
& +\quad C_{1} \frac{C_{\delta}}{\varepsilon^{1+3 / \delta}}\left|z_{1}-z_{2}\right|_{X_{t}}^{2}|v|_{\left.L^{2+6 / \delta_{0}\left(0, t ; \mathbb{L}^{3+\delta_{0}}(D)\right)}\right) .} .
\end{aligned}
$$

Now let us choose $\varepsilon>0$ that $C_{1} \varepsilon^{1+\delta_{0} / 3}=1 / 2$ and denote $K=C_{1} \frac{C_{\delta}}{\varepsilon^{1+3 / \delta}}$. We have

(5.11) $\left\|\Phi_{t}\left(z_{1}\right)-\Phi_{t}\left(z_{2}\right)\right\|_{X_{t}}^{2} \leq\left(1 / 2+K|v|_{L^{2+6 / \delta_{0}}\left(0, t ; \mathbb{L}^{\left.3+\delta_{0}(D)\right)}\right.}\right)\left|z_{1}-z_{2}\right|_{X_{t}}^{2}, t \in[0, T]$.

Choose $t=T_{1}$ such that $|v|_{L^{2+6 / \delta_{0}}\left(0, T_{1} ; \mathbb{L}^{3+\delta_{0}}(D)\right)} \leq d=\frac{1}{3 K}$ then $\Phi_{T_{1}}$ is an affine contraction map and by the Banach Fixed Point Theorem there exists a fixed point $F \in X_{T_{1}}$ of $\Phi_{T_{1}}$. Obviously $F$ is a solution of problem (3.1-3.2) on interval [0, $\left.T_{1}\right]$.

Existence of a global solution. From Lemma 5.1 and assumption (3.5) it follows that we can find partition $0=T_{0}<T_{1}<\ldots<T_{k-1}<T_{k}=T$ of interval $[0, T]$ such that $|v|_{L^{2+6 / \delta_{0}}\left(T_{i}, T_{i+1} ; \mathbb{L}^{3+\delta_{0}}(D)\right)}<\frac{1}{3 K}, i=0, \ldots, k-1$. Therefore, we can use the inequality (5.11) and the Banach Fixed Point Theorem iteratively to define global solution.

(iii) To proof the statement in part [iii] we will use a method suggested by Temam in [34]. We will consider only the case $k=1$. General case follows by induction. Let us recall that

$$
A(t)=\nu A+B(v(t), \cdot) \text {. }
$$

By differentiating the equation (3.1-3.2) w.r.t. $t$ (in weak sense) we find that $F^{\prime}$ is a solution of

$$
\frac{d F^{\prime}}{d t}=-A(t) F^{\prime}+B\left(v^{\prime}(t), F\right)+f^{\prime}, t \in[0, T]
$$

Now from the assumptions of the statement in part [ii] it follows that it is enough to prove that $B\left(v^{\prime}(\cdot), F\right) \in L^{2}(0, T ; H)$ and then use the already proven statement in part [i]. From inequality (2.20) we have

$$
\begin{aligned}
& \int_{0}^{T}\left|B\left(v^{\prime}(t), F\right)\right|_{H}^{2} d t \\
\leq & \varepsilon^{1+\delta_{0} / 3} \int_{0}^{T}\|\operatorname{curl} F\|_{V}^{2} d t+\frac{C_{\delta_{0}}}{\varepsilon^{1+3 / \delta_{0}}} \int_{0}^{T}\left|v^{\prime}(t)\right|_{\mathbb{L}^{3+\delta_{0}(D)}}^{2}|\operatorname{curl} F|_{H}^{2} d t \\
\leq & \varepsilon^{1+\delta_{0} / 3}|F|_{L^{2}(0, T ; D(A))}+\frac{C_{\delta_{0}}}{\varepsilon^{1+3 / \delta_{0}}}\|F\|_{C([0, T] ; V)}^{2} \int_{0}^{T}\left|v^{\prime}(t)\right|_{\mathbb{L}^{3+\delta_{0}(D)}}^{2+\frac{6}{\delta_{0}}} d t<\infty .
\end{aligned}
$$


Note that $F \in C([0, T] ; V)$ by Lemma 2.1 .

PROOF OF PROPOSITION 3.4. The proof is very similar to the proof of the previous Proposition.

(i) The proof will be divided into three parts a), b), c).

a) First we consider a special case when $v \in L^{\infty}\left(0, T ; \mathbb{L}^{3+\delta_{0}}(D)\right)$. We will use Theorem 2.3 with Gelfand triple $V \subset H \cong H^{\prime} \subset V^{\prime}$. Denote $B(t)=\nu A+\operatorname{curl}(v(t) \times \cdot)$. We need to check whether the conditions (2.5) and (2.6) are satisfied. We have

$$
\begin{aligned}
\langle B(t) f, f\rangle_{V^{\prime}, V} & =\nu \tilde{a}(f, f)+\langle\operatorname{curl}(v(t) \times f), f\rangle_{V^{\prime}, V} \\
& =\nu \tilde{a}(f, f)+\langle v(t) \times f, \operatorname{curl} f\rangle_{V^{\prime}, V}, t \in[0, T], f \in V .
\end{aligned}
$$

Now we can use the inequality (5.2) and the continuity of the form $\tilde{a}$ to get

$$
\|B(t)\|_{\mathcal{L}\left(V, V^{\prime}\right)} \leq C \nu+C_{2}|v(t)|_{\mathbb{L}^{3+\delta_{0}}(D)} .
$$

The coercivity condition (2.6) can be proved in the same way as in the proof of Proposition 3.2. Therefore, by Theorem 2.3 first statement of the Proposition is proved in our special case.

b) To prove Proposition in the general case we will, as before, show an energy inequality for solutions of the problem (3.3-3.4) when $v \in L^{\infty}\left(0, T ; \mathbb{L}^{3+\delta_{0}}(D)\right)$. From Step (a) we know that there exists a unique solution $G \in L^{2}(0, T ; V)$ such that $G^{\prime} \in L^{2}\left(0, T ; V^{\prime}\right)$. Then, from Lemma 2.1 it follows that $G \in C([0, T] ; H)$ and

$$
\begin{aligned}
\frac{1}{2} \frac{d}{d t}|G|_{H}^{2} & =-\nu\|G\|_{V}^{2}+\langle f, G\rangle_{V^{\prime}, V}-\langle v \times G, \operatorname{curl} G\rangle_{V^{\prime}, V} \\
& =-\nu\|G\|_{V}^{2}+\langle f, G\rangle_{V^{\prime}, V}+(\operatorname{curl} G, v \times G)_{H}
\end{aligned}
$$

Therefore, by the Young inequality,

$$
\begin{aligned}
|G(t)|_{H}^{2} & +2 \nu \int_{0}^{t}|G(s)|_{V}^{2} d s-\int_{0}^{t}(\operatorname{curl} G(s), v(s) \times G(s))_{H} d s \\
& =|G(0)|_{H}^{2}+\int_{0}^{t}\langle f(s), G(s)\rangle_{V^{\prime}, V} d s \\
& \leq|G(0)|_{H}^{2}+\frac{\nu}{2} \int_{0}^{t}|G(s)|_{V}^{2} d s+\frac{C}{\nu} \int_{0}^{t}|f(s)|_{V^{\prime}}^{2} d s, t \in[0, T] .
\end{aligned}
$$

The term $\int_{0}^{t}(\operatorname{curl} G(s), v(s) \times G(s))_{H} d s$ can be estimated in the same way as in Proposition 3.2, see (5.4). Thus we infer that $G$ satisfies the following inequality, for $t \in[0, T]$,

$$
|G(t)|_{H}^{2}+\nu \int_{0}^{t}\|G(s)\|_{V}^{2} d s \leq K_{1}\left(|G(0)|_{H}^{2}+\frac{C}{\nu} \int_{0}^{t}|f(s)|_{V^{\prime}}^{2} d s\right)
$$

$$
\left(1+\int_{0}^{t}|v(s)|_{\mathbb{L}^{3+\delta_{0}}}^{2+6 / \delta_{0}} d s\right) e^{C\left(\delta_{0}, \nu\right) \int_{0}^{t}|v(s)|_{\mathbb{L}^{3+\delta_{0}}}^{2+6 / \delta_{0}} d s}
$$


c) The general case. Now, let $\left\{v_{n}\right\}_{n=1}^{\infty}$ be an $L^{\infty}\left(0, T ; \mathbb{L}^{3+\delta_{0}}(D)\right)$-valued sequence of functions such that $v_{n} \rightarrow v \in L^{2+\frac{6}{\delta_{0}}}\left(0, T ; \mathbb{L}^{3+\delta_{0}}(D)\right), n \rightarrow \infty$ in $L^{2+\frac{6}{\delta_{0}}}\left(0, T ; \mathbb{L}^{3+\delta_{0}}(D)\right)$. Let $\left\{G_{n}\right\}_{n=1}^{\infty}$ be corresponding sequence of solutions of the problem (3.3-3.4). Then from (5.13) it follows that sequence $\left\{G_{n}\right\}_{n=1}^{\infty}$ lie in a bounded set of $L^{\infty}(0, T ; H) \cap L^{2}(0, T ; V)$. Using the same argument as in the proof of Proposition 3.2 we can find subsequence $\left\{G_{n^{\prime}}\right\}_{n^{\prime}=1}^{\infty}$ weakly convergent to $G \in L^{\infty}(0, T ; H) \cap L^{2}(0, T ; V)$ which solves the problem (3.3-3.4) in a weak sense. Moreover, it follows from inequality (5.13), that the function $G$ satisfies energy inequality (3.9). Uniqueness of the solution of the problem (3.3-3.4) follows from the energy inequality (3.9). The only difference with the previous Proposition is that now we can prove that $G^{\prime} \in L^{2}\left(0, T, V^{\prime}\right)$. Indeed, we have

$$
\begin{aligned}
\left\|G^{\prime}\right\|_{L^{2}\left(0, T, V^{\prime}\right)}^{2} & =\|B G\|_{L^{2}\left(0, T, V^{\prime}\right)}^{2} \\
& \leq \int_{0}^{T}|\nu A G+\operatorname{curl}(v(t) \times G(t))|_{V^{\prime}}^{2} d t \\
& \leq \nu^{2}\|G\|_{L^{2}(0, T, V)}^{2}+\int_{0}^{T}|v(t) \times G(t)|_{H}^{2} d t \\
& \leq \nu^{2}\|G\|_{L^{2}(0, T, V)}^{2}+\int_{0}^{T}\left(C_{1}|G(t)|_{V}^{2}+C_{2}|v(t)|_{\mathbb{L}^{3+\delta_{0}}}^{2+6 / \delta_{0}}|G(t)|_{H}^{2}\right) d t \\
& \leq C_{3}\|G\|_{L^{2}(0, T, V)}^{2}+C_{2}\|G\|_{L^{\infty}(0, T, H)}^{2}|v|_{L^{2+\frac{6}{\delta_{0}}}}{ }_{\left(0, T ; \mathbb{L}^{\left.3+\delta_{0}(D)\right)}\right.}<\infty .
\end{aligned}
$$

Thus, the first statement of Proposition is proved. Statements [ii] and [iii] can be proved in the same way as in the proof of Proposition 3.2.

(ii) Existence of a local solution. Let $X_{T}=\left\{F \in L^{2}(0, T ; D(A)): F^{\prime} \in L^{2}(0, T ; H)\right\}$ be a Banach space endowed with a norm

$$
|F|_{X_{T}}^{2}=\nu^{2}|F|_{L^{2}(0, T ; D(A))}^{2}+\left|F^{\prime}\right|_{L^{2}(0, T ; H)}^{2} .
$$

We will prove the following result.

Lemma 5.3. If $v \in L^{2}(0, T ; V), z \in X_{T}$ then $\operatorname{curl}(v(t) \times z) \in L^{2}(0, T ; H)$.

In view of Proposition 2.4 and the above Lemma, a map $\Phi_{T}: X_{T} \rightarrow X_{T}$ defined by $\Phi_{T}(z)=G$ iff $G$ is the unique solution of the problem

$$
G^{\prime}+\nu A G=f-\operatorname{curl}(v(t) \times z), G(0)=F_{0} \in V
$$

is well defined.

Proof of Lemma 5.3. We have:

$$
\begin{aligned}
\|\operatorname{curl}(v(t) \times z)\|_{L^{2}(0, T ; H)}^{2} & \leq C\left(\|z \nabla v\|_{L^{2}(0, T ; H)}^{2}+\|v \nabla z\|_{L^{2}(0, T ; H)}^{2}\right) \\
& \leq C|z|_{C([0, T] ; V)}|v|_{L^{2}(0, T ; V)}
\end{aligned}
$$

and the result follows from Lemma 2.1.

Now we will show that there exists $T_{1} \in(0, T]$ such that $\Phi_{T_{1}}$ is a strict contraction. Let us fix $t \in(0, T]$ and take $z_{1}, z_{2} \in X_{T}$. Then, by Proposition 2.4 and Lemma 2.12, we 
have

$$
\begin{aligned}
\left\|\Phi_{t}\left(z_{1}\right)-\Phi_{t}\left(z_{2}\right)\right\|_{X_{t}}^{2} & \leq C_{1}\left\|\operatorname{curl}\left(v(t) \times\left(z_{1}-z_{2}\right)\right)\right\|_{L^{2}(0, t ; H)}^{2} \\
& \leq C\left|z_{1}-z_{2}\right|_{C(0, t ; V)}^{2}|v|_{L^{2}(0, t ; V)}^{2} \leq C\left|z_{1}-z_{2}\right|_{X_{t}}^{2}|v|_{L^{2}(0, t ; V)}^{2} .
\end{aligned}
$$

Let us choose $T_{1} \in(0, T]$ such that $C|v|_{L^{2}\left(0, T_{1} ; V\right)}<1 / 2$. Then $\Phi_{T_{1}}$ is a strict contraction map and hence by the Banach Fixed Point Theorem there exists a unique $F \in X_{T_{1}}$ that is a fixed point of $\Phi_{T_{1}}$. By the definition of the $\Phi_{T}$ it follows that $F \in X_{T_{1}}$ is a solution of problem (3.1-3.2) on interval $\left[0, T_{1}\right]$. Notice also that $F\left(T_{1}\right) \in V$. Therefore, the map $\Phi_{T}$ with initial data $F\left(T_{1}\right)$ is well defined on interval $\left[T_{1}, T\right]$.

Existence of a global solution. From Lemma 5.1 and assumption (3.5) it follows that we can find a partition $0=T_{0}<T_{1}<\ldots<T_{k-1}<T_{k}=T$ of the interval $[0, T]$ such that $|v|_{L^{2}\left(T_{i}, T_{i+1} ; V\right)}<1 / 2, i=0, \ldots, k-1$. Therefore, we can use inequality (5.11) and the Banach Fixed Point Theorem iteratively to define a global solution (3.3-3.4).

(iii) We will consider only the case $k=1$. General case follows by induction. We differentiate equation (3.3-3.4) w.r.t. $t$ (in the weak sense) and get an equation for the function $G^{\prime}$ :

$$
\begin{aligned}
\frac{\partial G^{\prime}}{\partial t}(t) & =-\nu A G^{\prime}(t)-\operatorname{curl} G^{\prime}(t) \times v(t)-\operatorname{curl}\left(G(t) \times v^{\prime}(t)\right)+f^{\prime}(t) \\
G^{\prime}(0) & =-\nu A G_{0}-\operatorname{curl} v(0) \times G_{0}+f(0), t \in[0, T]
\end{aligned}
$$

Now from the assumptions of the statement [ii] it follows that it is enough to prove that $\operatorname{curl}\left(G \times v^{\prime}(t)\right) \in L^{2}(0, T ; H)$ and then use the already proven statement in part [i]. By the inequality (5.15) we have

$$
\left\|\operatorname{curl}\left(G \times v^{\prime}\right)\right\|_{L^{2}(0, T ; H)}^{2} \leq C|G|_{C([0, T] ; V)}\left|v^{\prime}\right|_{L^{2}(0, T ; V)}<\infty
$$

Note that $G \in C([0, T] ; V)$ by Lemma 2.1 .

Proof of Theorem 3.7. $1^{\text {st }}$ Step. Fix $\delta_{0}>0$. Let us prove the Theorem in the case of smooth initial data $F_{0} \in C^{\infty}(\bar{D}) \cap H$ and vector field $v \in C_{b}^{\infty}([0, T] \times \bar{D}) \cap$ $L^{2+\frac{6}{\delta_{0}}}\left(0, T ; \mathbb{L}^{3+\delta_{0}}(D)\right)$. For each $\varepsilon>0$ we can find $F_{0}^{\varepsilon} \in C^{\infty}(\bar{D}) \cap H, G_{0}^{\varepsilon} \in C^{\infty}(\bar{D}) \cap$ $H, v^{\varepsilon} \in C_{b}^{\infty}([0, T] \times \bar{D}) \cap L^{2+\frac{6}{\delta_{0}}}\left(0, T ; \mathbb{L}^{3+\delta_{0}}(D)\right)$ such that as $\varepsilon \rightarrow 0, F_{0}^{\varepsilon} \rightarrow F_{0}$ in $H, G_{0}^{\varepsilon} \rightarrow G_{0}$ in $H$ and $v^{\varepsilon} \rightarrow v$ in $L^{\infty}\left(0, T ; \mathbb{L}^{3+\delta_{0}}(D)\right)$. It follows from Corollaries 3.5 and 3.6 that there exists $F^{\varepsilon} \in C([0, T] ; H) \cap C^{\infty}((0, T] \times \bar{D}), G^{\varepsilon} \in C([0, T] ; H) \cap$ $\left.C^{\infty}((0, T] \times \bar{D})\right)$ that are solutions to the following problems

$$
\begin{aligned}
\frac{\partial F^{\varepsilon}}{\partial t}(t) & =-\nu A F^{\varepsilon}(t)-P\left(v^{\varepsilon}(t) \times \operatorname{curl} F^{\varepsilon}(t)\right) \\
F^{\varepsilon}(0, \cdot) & =F_{0}^{\varepsilon}, t \in[0, T] \\
\frac{\partial G^{\varepsilon}}{\partial t}(t) & =-\nu A G^{\varepsilon}(t)+\operatorname{curl}\left(v^{\varepsilon}(T-t) \times G^{\varepsilon}(t)\right) \\
G^{\varepsilon}(0, \cdot) & =G_{0}^{\varepsilon}, t \in[0, T]
\end{aligned}
$$


Therefore, for all $t \in(0, T]$ we have

$$
\begin{aligned}
& \frac{d}{d t}\left(F^{\varepsilon}(t), G^{\varepsilon}(T-t)\right)_{\mathbb{L}^{2}(D)} \\
= & \left(\frac{d}{d t} F^{\varepsilon}(t), G^{\varepsilon}(T-t)\right)_{\mathbb{L}^{2}(D)}-\left(F^{\varepsilon}(t), \frac{d}{d t} G^{\varepsilon}(T-t)\right)_{\mathbb{L}^{2}(D)} \\
= & \nu\left(\mathrm{P} \triangle F^{\varepsilon}(t), G^{\varepsilon}(T-t)\right)_{\mathbb{L}^{2}(D)}-\left(\mathrm{P}\left(v(t) \times \operatorname{curl} F^{\varepsilon}(t)\right), G^{\varepsilon}(T-t)\right)_{\mathbb{L}^{2}(D)} \\
- & \nu\left(F^{\varepsilon}(t), \mathrm{P} \triangle G^{\varepsilon}(T-t)\right)_{\mathbb{L}^{2}(D)}-\left(F^{\varepsilon}(t), \operatorname{curl}\left(v(t) \times G^{\varepsilon}(T-t)\right)\right)_{\mathbb{L}^{2}(D)} \\
= & K_{1}(t)-K_{2}(t)-K_{3}(t)-K_{4}(t)
\end{aligned}
$$

It follows from the fact that $\operatorname{div} F^{\varepsilon}=\operatorname{div} G^{\varepsilon}=0,\left.F^{\varepsilon}\right|_{\partial D}=\left.G^{\varepsilon}\right|_{\partial D}=0$ and the integration by parts formula that $\left(F^{\varepsilon}, \nabla \psi\right)_{\mathbb{L}^{2}(D)}=\left(G^{\varepsilon}, \nabla \psi\right)_{\mathbb{L}^{2}(D)}=0$ for any $\psi \in C^{\infty}(\bar{D})$. Thus, we have

$$
\begin{aligned}
K_{1}(t) & =\left(\mathrm{P} \triangle F^{\varepsilon}(t), G^{\varepsilon}(T-t)\right)_{\mathbb{L}^{2}(D)}=\left(\triangle F^{\varepsilon}(t), G^{\varepsilon}(T-t)\right)_{\mathbb{L}^{2}(D)}, \\
K_{2}(t) & =\left(\mathrm{P}\left(v(t) \times \operatorname{curl} F^{\varepsilon}(t)\right), G^{\varepsilon}(T-t)\right)_{\mathbb{L}^{2}(D)} \\
& =\left(v(t) \times \operatorname{curl} F^{\varepsilon}(t), G^{\varepsilon}(T-t)\right)_{\mathbb{L}^{2}(D)}, t \in(0, T]
\end{aligned}
$$

and

$$
K_{3}(t)=\left(F^{\varepsilon}(t), \mathrm{P} \triangle G^{\varepsilon}(T-t)\right)_{\mathbb{L}^{2}(D)}=\left(F^{\varepsilon}(t), \triangle G^{\varepsilon}(T-t)\right)_{\mathbb{L}^{2}(D)}, t \in(0, T]
$$

Therefore, by the Green Formula we get $K_{1}(t)-K_{3}(t)=0, t \in(0, T]$. From (2.1), (5.16) and the formula

$$
\int_{D} u \operatorname{curl} v d x-\int_{D} v \operatorname{curl} u d x=\int_{\partial D}(u \times v, \vec{n}) d \sigma
$$

we infer that

$$
\begin{aligned}
K_{2}(t) & =\left(v(t) \times \operatorname{curl} F^{\varepsilon}(t), G^{\varepsilon}(T-t)\right)_{\mathbb{L}^{2}(D)} \\
& =-\left(\operatorname{curl} F^{\varepsilon}(t) \times v(t), G^{\varepsilon}(T-t)\right)_{\mathbb{L}^{2}(D)} \\
& =-\left(\operatorname{curl} F^{\varepsilon}(t), v(t) \times G^{\varepsilon}(T-t)\right)_{\mathbb{L}^{2}(D)}=-K_{4}(t), t \in(0, T] .
\end{aligned}
$$

Thus, $\frac{d}{d t}\left(F^{\varepsilon}(t), G^{\varepsilon}(T-t)\right)_{\mathbb{L}^{2}(D)}=0, t \in(0, T]$. Also, by the regularity of $F^{\varepsilon}, G^{\varepsilon}$ it follows that $\left(F^{\varepsilon}(\cdot), G^{\varepsilon}(T-\cdot)\right)_{\mathbb{L}^{2}(D)} \in C^{\infty}((0, T]) \cap C([0, T])$. As a result we get equality (3.13).

$2^{\text {nd }}$ step. Let us suppose that we have showed that for each $t \in[0, T], F_{\varepsilon}(t) \rightarrow F(t)$ in weak topology of $H$ and that $G_{\varepsilon} \rightarrow G$ in $C([0, T], H)$. Then we have

$$
\begin{aligned}
& \left|(F(t), G(T-t))-\left(F^{\varepsilon}(t), G^{\varepsilon}(T-t)\right)\right| \\
= & \left|\left(F-F^{\varepsilon}(t), G(T-t)\right)+\left(F^{\varepsilon}(t), G-G^{\varepsilon}(T-t)\right)\right| \\
\leq & \left|\left(F-F^{\varepsilon}(t), G(T-t)\right)\right|+\left|F^{\varepsilon}(t)\right|_{H}\left|G-G^{\varepsilon}(T-t)\right|_{H} \\
\leq & \left|\left(F-F^{\varepsilon}(t), G(T-t)\right)\right|+\left|F_{0}^{\varepsilon}\right|_{H} \sup _{s \in[0, T]}\left|G-G^{\varepsilon}(s)\right|_{H} \stackrel{\varepsilon \rightarrow 0}{\rightarrow} 0, t \in[0, T]
\end{aligned}
$$

i.e. $(F(t), G(T-t))_{H}=\lim _{\varepsilon \rightarrow 0}\left(F^{\varepsilon}(t), G^{\varepsilon}(T-t)\right)_{H}, t \in[0, T]$ and the result follows from first step. In order to show weak convergence of $F_{\varepsilon}(t)$ to $F(t), t \in[0, T]$ let us first notice that by the Banach-Alaoglu Theorem, $F_{\varepsilon}$ converges to $F$ weakly-* in $L^{\infty}(0, T ; H)$. The proof of this claim can be performed in exactly the same manner as the proof of the convergence of $F_{n} \rightarrow F$ in Proposition 3.2). Also, we have from the Banach-Alaoglu Theorem that $F^{\varepsilon}(t)$ weakly-* convergent to some $\Psi(t) \in H, t \in[0, T]$. We will show that $\Psi=F$. Fix $\xi \in V$. Let us denote $g(t)=(\Psi(t)-F(t), \xi)_{H}, t \in[0, T]$. Since $V$ is dense 
in $H$ it is enough to show that $g=0$. Now we will show that $g \in C([0, T])$. From the part (i) of Proposition 3.2 we infer that $F \in C\left([0, T], V^{\prime}\right)$. Thus, $(F(\cdot), \xi)_{H}=\langle F(\cdot), \xi\rangle_{V^{\prime}, V} \in$ $C([0, T])$. Furthermore, for $t \in[0, T]$ we have

$$
\begin{aligned}
& \left|\left(F_{\varepsilon}(t), \xi\right)-\left(F_{\varepsilon}(s), \xi\right)\right| \leq \int_{s}^{t}\left|\left\langle F_{\varepsilon}^{\prime}(r), \xi\right\rangle\right| d r \\
\leq & \left(\int_{0}^{T}\left|\left\langle F_{\varepsilon}^{\prime}(r), \xi\right\rangle\right|^{1+\frac{3}{2 \delta_{0}+3}} d r\right)^{\frac{2 \delta_{0}+3}{2 \delta_{0}+6}}|t-s|^{\frac{3}{2 \delta_{0}+6}} \\
\leq & \left|F_{\varepsilon}^{\prime}\right|_{L^{1+\frac{3}{2 \delta_{0}+3}}\left(0, T ; V^{\prime}\right)}|\xi|_{V}|t-s|^{\frac{3}{2 \delta_{0}+6}} \\
\leq & C\left|F_{\varepsilon}\right|_{L^{2}(0, T ; V)}\left(C_{1}\left(\nu, T, \delta_{0}\right)+\left|v_{\varepsilon}\right|_{L^{2+\frac{6}{2 \delta_{0}+6}}}^{\frac{2}{\delta_{0}+3}}\left(0, T ; \mathbb{L}^{3+\delta_{0}}(D)\right)\right. \\
\leq & )^{\frac{2 \delta_{0}+3}{2 \delta_{0}+6}}|\xi|_{V}|t-s|^{\frac{3}{2_{0}+6}} \\
\leq & C\left|F_{\varepsilon}^{0}\right|_{H}\left(C\left(\nu, T, \delta_{0}\right)+\left|v_{\varepsilon}\right|_{L^{2+\frac{6}{\delta_{0}}}\left(0, T ; \mathbb{L}^{3+\delta_{0}}(D)\right)}\right)|\xi|_{V}|t-s|^{\frac{3}{2 \delta_{0}+6}} \\
\leq & C\left|F^{0}\right|_{H}\left(C\left(\nu, T, \delta_{0}\right)+|v|_{L^{2+\frac{6}{\delta_{0}}}\left(0, T ; \mathbb{L}^{3+\delta_{0}}(D)\right)}\right)|\xi|_{V}|t-s|^{\frac{3}{2^{2}+6}}, 0<s \leq t<T .
\end{aligned}
$$

In the above sequence of inequalities, the first one follows because $\left(F_{\varepsilon}(\cdot), \xi\right) \in$ $C^{\infty}((0, T))$, the second one from the Hölder inequality and the fourth one from the inequality (5.9).

Taking the $\varepsilon \searrow 0$ limit in (5.17) we immediately get

$$
|(\Psi(t), \xi)-(\Psi(s), \xi)| \leq C\left(F_{0}, v, \nu, \delta_{0}, T\right)|\xi|_{V}|t-s|^{\frac{3}{2 \delta_{0}+6}},
$$

where $C\left(F_{0}, v, \nu, \delta_{0}, T\right)=C\left|F^{0}\right|_{H}\left(C\left(\nu, T, \delta_{0}\right)+|v|_{L^{2+\frac{6}{\delta_{0}}}\left(0, T ; \mathbb{L}^{3+\delta_{0}}(D)\right)}\right)$. Hence, $\Psi \in$ $C\left([0, T], V^{\prime}\right)$ and, consequently, $g \in C([0, T])$. Therefore it is enough to prove that $g(t)=$ 0 for a.a. $t \in[0, T]$.

We have already observed that

$$
\lim _{\varepsilon \searrow 0} \int_{0}^{T}\left(F^{\varepsilon}(s)-F(s), q(s)\right)_{H} d s=0, \text { for all } q \in L^{1}(0, T ; H) .
$$

Let us take any $f \in L^{1}(0, T)$ and put $q=\xi f, g_{\varepsilon}=\left(F^{\varepsilon}(\cdot)-F(\cdot), \xi\right)_{H}$. Then from (5.19) we infer that

$$
\int_{0}^{T} g_{\varepsilon}(s) f(s) d s \rightarrow 0, \text { for all } f \in L^{1}(0, T) .
$$

On the other hand, it follows from the definition of $g$ that $g_{\varepsilon} \rightarrow g$ pointwise. We will show now that (5.20) and this pointwise convergence of $g_{\varepsilon}$ imply that $g=0$ a.e.. Indeed, by the Egorov Theorem, see e.g. [1, Theorem 2.2.1, p. 110], for any $l>0$ there exists a measurable set $A_{l} \subset[0, T]$ such that $\lambda\left(A_{l}\right)<l$ and $g_{\varepsilon} \rightarrow g$ uniformly on $[0, T] \backslash A_{l}$. Here $\lambda$ denotes the Lebesgue measure. Hence by (5.20) we infer that $g(t)=0$, for a.e. $t \in[0, T] \backslash A_{l}$ and consequently $g(t)=0$ for a.e. $t \in[0, T]$. 
Thus, it remains to show that $G_{\varepsilon} \rightarrow G$ in $C([0, T], H)$. Denote $R^{\varepsilon}=G^{\varepsilon}-G$. Then $R^{\varepsilon}$ is a solution to the following problem.

$$
\begin{aligned}
& \frac{\partial R^{\varepsilon}}{\partial t}(t)=-\nu A R^{\varepsilon}(t) \\
& +\operatorname{curl}\left(v^{\varepsilon}(T-t) \times R^{\varepsilon}(t)\right)+\operatorname{curl}\left(\left(v^{\varepsilon}(T-t)-v(T-t)\right) \times G(t)\right) \\
& R^{\varepsilon}(0, \cdot)=G_{0}^{\varepsilon}-G_{0}, t \in[0, T] .
\end{aligned}
$$

Applying the energy inequality (3.9) to the function $R^{\varepsilon}$ we infer that for any $\tau>0$

$$
\begin{aligned}
& \left|R^{\varepsilon}\right|_{C([0, T] ; H)}^{2} \leq C\left(\left|v^{\varepsilon}\right|_{L^{2+\frac{6}{\delta_{0}}}\left(0, T ; \mathbb{L}^{\left.3+\delta_{0}(D)\right)}\right.}\right)\left(\left|G_{0}^{\varepsilon}-G_{0}\right|_{H}^{2}\right. \\
& \left.+\left|\operatorname{curl}\left(\left(v-v^{\varepsilon}\right) \times G\right)\right|_{L^{2}\left(0, T ; V^{\prime}\right)}^{2}\right) \\
\leq & C\left(\left|v^{\varepsilon}\right|_{L^{2+\frac{6}{\delta_{0}}}\left(0, T ; \mathbb{L}^{\left.3+\delta_{0}(D)\right)}\right.}\right)\left(\left|G_{0}^{\varepsilon}-G_{0}\right|_{H}^{2}+\left|\left(v-v^{\varepsilon}\right) \times G\right|_{L^{2}(0, T ; H)}^{2}\right) \\
\leq & C\left(|v|_{L^{2+\frac{6}{\delta_{0}}}\left(0, T ; \mathbb{L}^{\left.3+\delta_{0}(D)\right)}\right.}\right)\left(\left|G_{0}^{\varepsilon}-G_{0}\right|_{H}^{2}+\tau^{1+\delta_{0} / 3} \int_{0}^{T}|G(s)|_{V}^{2} d s+\right. \\
& \left.\frac{C_{\delta_{0}}}{\tau^{1+3 / \delta_{0}}}|G|_{C([0, T] ; H)}^{2}\left|v^{\varepsilon}-v\right|_{L^{2+\frac{6}{\delta_{0}}}\left(0, T ; \mathbb{L}^{\left.3+\delta_{0}(D)\right)}\right.}\right),
\end{aligned}
$$

where last inequality of (5.21) follows from Lemma 2.12. Now, from the convergences $v^{\varepsilon} \rightarrow v$ in $L^{2+\frac{6}{\delta_{0}}}\left(0, T ; \mathbb{L}^{3+\delta_{0}}(D)\right), G_{0}^{\varepsilon} \rightarrow G_{0}$ in $H$ and inequalities (5.21) we get the result.

\section{References}

[1] V.I. Bogachev, Measure theory. Vol. I, Springer-Verlag, Berlin, 2007.

[2] F. Bouchut, F. James, S. Mancini, Uniqueness and weak stability for multi-dimensional transport equations with one-sided Lipschitz coefficient, Ann. Sc. Norm. Super. Pisa Cl. Sci. (5) 4 (2005), no. 1, 1-25.

[3] Z. Brzeźniak, On analytic dependence of solutions of Navier-Stokes equations with respect to exterior force and initial velocity, Univ. Iagel. Acta Math. No. 28 (1991), 111-124.

[4] Z. Brzeźniak and Y. Li, Asymptotic compactness and absorbing sets for $2 D$ Stochastic Navier-Stokes equations on some unbounded domains, Trans. Amer. Math. Soc. 358 (2006), no. 12, 5587-5629.

[5] B. Busnello, F. Flandoli, M. Romito, A probabilistic representation for the vorticity of a three-dimensional viscous fluid and for general systems of parabolic equations, Proc. Edinb. Math. Soc. (2) 48 (2005), no. 2, 295-336.

[6] P. Constantin , G. Iyer, A stochastic Lagrangian representation of the three-dimensional incompressible Navier-Stokes equations, Comm. Pure and Applied Mathematics, 61 (2008), no. 3, 330-345.

[7] R. J. DiPerna, P.-L. Lions, Ordinary differential equations, transport theory and Sobolev spaces, Invent. Math. 98 (1989), no. 3, 511-547.

[8] K. Engø, On the BCH-formula in $\mathfrak{s o ( 3 ) , ~ B I T . ~} 41$ (2001), no. 3, 629-632.

[9] G. Falkovich, K. Gawedzki, M. Vergassola, Particles and fields in fluid turbulence, Rev. Mod. Phys. 73 (2001), 913-975.

[10] A. Friedman, Partial differential equations, Holt, Rinehart and Winston Inc., New York-Montreal, Que.-London, 1969.

[11] D. Fujiwara, H. Morimoto, An $L_{r}$-theorem of the Helmholtz decomposition of vector fields. J. Fac. Sci. Univ. Tokyo Sect. IA Math. 24 (1977), no. 3, 685-700.

[12] L. Hörmander, The Analysis of Linear Partial Differential Operators I, 2nd ed., Springer, Berlin, 1990.

[13] N. Ikeda and S. Watanabe, Stochastic Differential Equations AND Diffusion Proces SES, John Wiley and Sons, New York, 1981.

[14] A. N. Kolmogorov, S. V. Fomin, ELEMENTS of THE THEORY OF FUNCTIONS AND FUNCTIONAL ANALYSIS(in russian), Nauka, Moscow, 4th edition, 1976. 
[15] N. V. Krylov, On Kolmogorovs equations for finite dimensional diffusions, in Stochastic PDEs and Kolmogorov equations in infinite dimensions (ed. G. Da Prato), Lecture Notes in Mathematics, vol. 1715. Springer, 1999

[16] H. Kunita, Stochastic flows and stochastic Differential EQuations, Cambridge Studies in Advanced Mathematics, 24. Cambridge University Press, Cambridge, 1990.

[17] O. A. Ladyzhenskaya, On uniqueness and smoothness of generalized solutions to the Navier-Stokes equations, Zapiski Nauchn. Seminar. POMI, 5 (1967), 169-185.

[18] Y. Le Jan, O. Raimond, Integration of Brownian vector fields, Ann. Probab. 30 (2002), no. 2, 826-873.

[19] J.L. Lions and E. Magenes, NON-HOMOGENEOUS BOUNDARY VALUE PROBLEMS AND APPLICATIONS. VOL. I, Grundlehren der Mathematischen Wissenschaften [Fundamental Principles of Mathematical Sciences], 181. Springer-Verlag, New York-Heidelberg, 1972.

[20] J.L. Lions and E. Magenes, NON-HOMOgENEOUS Boundary VALUE PROBLEMS AND APPLICATIONS VOL. II, Grundlehren der Mathematischen Wissenschaften [Fundamental Principles of Mathematical Sciences], 182. Springer-Verlag, New York-Heidelberg, 1972.

[21] S. Maniglia, Probabilistic representation and uniqueness results for measure-valued solutions of transport equations, J. Math. Pures Appl. (9) 87 (2007), no. 6, 601-626.

[22] C. Marchioro, M. Pulvirenti, MAThematiCAL THEORY OF INCOMPRESSible NONVISCOUS FLUIDS, Applied Mathematical Sciences, 96. Springer-Verlag, New York, 1994.

[23] R. Mikulevicius, On the Cauchy problem for stochastic Stokes equations, SIAM J. Math. Anal., 34 (2002), 121-141.

[24] H. K. Moffat, The degree of knottedness of tangled vortex lines, J. Fluid Mech. 35 (1969), 117-129.

[25] M. Neklyudov, Controllable stochastic dynamical system equivalent to the Navier-Stokes equation, Russ. J. Math. Phys., 12 (2005), No. 2, 232-240.

[26] M. Neklyudov, Equivalence of Navier-Stokes equation and infinite dimensional Burgers equation(Russian), Fundamental and Applied Mathematics, 12 (2006), No. 5, 109-120; translated in Journal of Mathematical Sciences, 150 (2008), No. 6, 2531-2539.

[27] G. Prodi, Un teorema di unicitper le equazioni di Navier-Stokes, Ann. Mat. Pura Appl. (4) 48 (1959), 173182.

[28] W. Rudin, Real and COMPLEX Analysis, McGraw-Hill Book Co., New York, 1987

[29] J. Serrin, The initial Value Problem for the Navier-Stokes EQuations. Nonlinear ProbLEMS. Madison, Wis. pp. 69-98 Univ. of Wisconsin Press, Madison, Wis., 1963.

[30] M. Spivak, Calculus on manifolds. A MODERn APPROACH to ClassiCAl TheOREMS OF ADVANCED CAlCUlus. W. A. Benjamin, Inc., New York-Amsterdam, 1965.

[31] M. Spivak, A comprehensive introduction to differential Geometry. Vol. One. Published by M. Spivak, Brandeis Univ., Waltham, Mass. 1970.

[32] H. Tanabe, Equations of Evolution, Monographs and studies in mathematics, 6. Pitman Publishing Ltd., London, 1979.

[33] R.Temam, NAVier-Stokes Equations. Theory And numerical Analysis, AMS Chelsea Publishing, Providence, RI, 2001.

[34] R. Temam, Behaviour at time $t=0$ of the solutions of semilinear evolution equations,J. Differential Equations, 43 (1982), no. 1, 73-92.

[35] H. Triebel, Interpolation theORy, FUnCtion SPACES, DifFERENTIAL OPERATORS., North-Holland Publishing Co., Amsterdam-New York, 1978.

Department of Mathematics, The University of York, Heslington, York YO10 5DD, UK E-mail address: zb500@york. ac.uk

Department of Mathematics, The University of York, HeSLINGton, York YO10 5DD, UK E-mail address: mn505eyork.ac.uk 